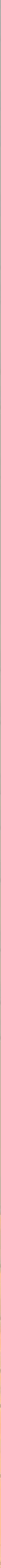




\title{
Inversion of adjunction for rational and Du Bois pairs
}

\author{
Sándor J. Kovács and Karl Schwede
}

\begin{abstract}
We prove several results about the behavior of Du Bois singularities and Du Bois pairs in families. Some of these generalize existing statements about Du Bois singularities to the pair setting while others are new even in the nonpair setting. We also prove a new inversion of adjunction result for Du Bois and rational pairs. In the nonpair setting this asserts that if a family over a smooth base has a special fiber $X_{0}$ with Du Bois singularities and the general fiber has rational singularities, then the total space has rational singularities near $X_{0}$.
\end{abstract}

\section{Introduction}

Rational singularities have been the gold standard for "mild" singularities in algebraic geometry for several decades. Whenever a new class of varieties with singularities is discovered, the first question usually asked is whether or not the new varieties have rational singularities. A key reason for this is that varieties with rational singularities behave cohomologically as if they were smooth. However, for many purposes rational singularities are not broad enough. For instance, nodes are not rational singularities, and more generally, singularities appearing on stable varieties, that is, mild degenerations of smooth ones that are necessary to consider in order to compactify moduli spaces, are not always rational. The class of $\mathrm{Du}$ Bois (or DB) singularities is slightly more inclusive than rational singularities. Du Bois singularities behave cohomologically as if they had simple normal crossing singularities (i.e., a higher-dimensional version of nodes).

Recently, Kollár [2013] and Kovács [2011a] introduced the notions of rational and Du Bois pairs $(X, D)$ for a normal variety $X$ and a reduced divisor $D \subseteq X$. These notions are philosophically distinct from the singularities considered typically in the minimal model program since $(X, D)$ having rational (respectively Du

Kovács was supported in part by NSF grant DMS-0856185 and DMS-1301888 and the Craig McKibben and Sarah Merner Endowed Professorship in Mathematics at the University of Washington. Schwede was supported in part by NSF grant DMS-1064485, NSF FRG Grant DMS-1501115, NSF CAREER grant DMS-1501102 and a Sloan Fellowship.

MSC2010: primary 14J17; secondary 14E99, 14J10, 14D06, 14B05.

Keywords: Du Bois singularities, rational singularities, inversion of adjunction, vanishing theorems. 
Bois) singularities does not generally imply that the ambient space $X$ has rational (respectively Du Bois) singularities [Kollár 2013, Remark 2.81(2)] (respectively Examples 2.10, 2.14, and [Graf and Kovács 2014]). Instead the singularities of $(X, D)$ measure the connection between the singularities of $X$ and $D$ (a notion obviously connected with problems related to inversion of adjunction). Furthermore, like Du Bois singularities, if $(X, Z)$ is a Du Bois pair then the ideal sheaf of $Z$ satisfies various Kodaira-type vanishing theorems, an observation which we hope will be useful in the future.

Even though a priori rational and Du Bois singularities are not part of the class one usually associates with the minimal model program, these singularities play important roles in both the minimal model program and moduli theory via the fact that (semi)log canonical singularities are Du Bois [Kovács et al. 2010; Kollár and Kovács 2010]. In addition, Du Bois singularities have played important roles in various other contexts recently. They are arguably the largest class of singularities for which we know that Kodaira vanishing holds [Patakfalvi 2015], they appear in proofs of extension and other vanishing theorems [Greb et al. 2011], positivity theorems [Schumacher 2012], categorical resolutions [Lunts 2012], log canonical compactifications [Hacon and $\mathrm{Xu} 2013$ ] and many other results more directly related to the minimal model program.

It is now a basic tenet of the minimal model program that the right way to study singularities is via pairs; see [Kollár 1997; 2013]. This allows for more freedom in applications and makes inductive arguments easier. The same is true for rational and Du Bois singularities. The introduction of Du Bois pairs streamlined some existing proofs (see [Kollár 2013, Chapter 6]) and extended the realm of applications.

In this paper we extend several recent results on Du Bois singularities to the context of Du Bois pairs, notably the recent results on deformations of Du Bois singularities found in [Kovács and Schwede 2011a] and the requisite injectivity theorem, a result of Kollár and Kovács on the behavior of depth in Du Bois families, and the characterization of Cohen-Macaulay Du Bois singularities of [Kovács et al. 2010].

Furthermore, we prove a new inversion of adjunction statement for rational and Du Bois pairs. This statement is new even in the nonpair setting. Roughly speaking, in the nonpair setting it says that if $f: X \rightarrow B$ is a family over a smooth base such that the general fiber has rational singularities and the special fiber has Du Bois singularities, then $X$ has rational singularities in a neighborhood of the special fiber. See Theorem $\mathrm{E}$ below for the general statement.

We state each of these theorems below. We begin with the deformation statement. Theorem A (Theorem 4.2). Let $X$ be a reduced scheme essentially of finite type over $\mathbb{C}, Z \subseteq X$ a reduced subscheme and $H$ a reduced effective Cartier divisor on $X$ that does not contain any component of $Z$. If $(H, Z \cap H)$ is a Du Bois pair, then $(X, Z)$ is a Du Bois pair near $H$. 
Just as in the nonpair setting, to prove this we first show an injectivity theorem. Theorem B (Theorem 3.2). Let $X$ be a reduced scheme over $\mathbb{C}$ and $Z \subseteq X a$ reduced subscheme. Then the natural map

$$
\Phi^{j}: \mathcal{E} \chi t_{\mathscr{O}_{X}}^{j}\left(\underline{\Omega}_{X, Z}^{0}, \omega_{X}^{\cdot}\right) \hookrightarrow \mathcal{E} \chi t_{\mathscr{O}_{X}}^{j}\left(\mathscr{I}_{Z}, \omega_{X}^{\bullet}\right)
$$

is injective for every $j \in \mathbb{Z}$.

Here $\mathcal{E} \chi t_{\mathscr{O}_{X}}^{j}\left(\ldots, \omega_{X}^{\bullet}\right)$ is shorthand to denote $\boldsymbol{h}^{j}\left(\mathcal{R} \mathcal{H o m}_{\mathscr{O}_{X}}\left({ }_{-}, \omega_{X}^{\bullet}\right)\right)$.

We also generalize some of the results of [Kollár and Kovács 2010] for families to the context of Du Bois pairs.

Theorem C (Corollary 5.6). Let $f:(X, Z) \rightarrow B$ be a flat projective family with $\mathscr{O}_{Z}, \mathscr{I}_{Z}$ flat over $B$ as well. Assume that all the fiber pairs $\left(X_{b}, Z_{b}\right)$ are Du Bois. Assume also that $B$ is connected and the generic fibers $\left(\mathscr{I}_{Z}\right)_{\text {gen }}$ are Cohen-Macaulay. Then all the fibers $\left(\mathscr{I}_{Z}\right)_{b}$ are Cohen-Macaulay.

We have a multiplier ideal/module like characterization of Du Bois pairs.

Theorem $\mathbf{D}$ (Theorem 6.3). Let $X$ be a normal variety and $Z \subseteq X$ a divisor. Further, let $\pi: \widetilde{X} \rightarrow X$ be a log resolution of $(X, Z)$ with $E=\pi^{-1}(Z)_{\text {red }} \vee \operatorname{exc}(\pi)$. If $\mathscr{I}_{Z}$ is Cohen-Macaulay then $(X, Z)$ is Du Bois if and only if

$$
\pi_{*} \omega_{\widetilde{X}}(E) \simeq \omega_{X}(Z)
$$

All of the results above are used in the proof of our inversion of adjunction result.

Theorem $\mathbf{E}$ (Theorem 7.1). Let $f: X \rightarrow B$ be a flat projective geometrically integral family over a smooth connected base $B$ with $\operatorname{dim} B \geq 1, H=f^{-1}(0)$ the special fiber, and $D$ a reduced codimension-1 subscheme of $X$ which is flat over $B$. Assume that $\left(H,\left.D\right|_{H}\right)$ is a Du Bois pair and that $(X \backslash H, D \backslash H)$ is a rational pair. Then $(X, D)$ is a rational pair.

This last result is new even in the case $D=0$; see Corollary 7.8. In the special case when $X \backslash H$ is smooth and $D=0$, Theorem E follows from [Schwede 2007, Theorem 5.1].

Statements similar to Theorem $\mathrm{E}$ have been proved in many related situations. For instance, assume $D=0, X \backslash H$ is canonical and $H$ is semi-log canonical. Then it follows from inversion of adjunction that $X$ has canonical singularities, see [Kollár and Shepherd-Barron 1988, Theorem 5.1; Karu 2000, Theorem 2.5; Kawakita 2007]. A nonexhaustive list of some other related results includes [Fedder and Watanabe 1989, Proposition 2.13; Schwede 2009; Hacon 2014; Erickson 2014]. 


\section{Definitions and basic properties}

2A. Rational pairs. First we recall the notion of rational pairs defined by Kollár and Kovács, as described in [Kollár 2013, Chapter 2]. Note that a similar notion was defined by Schwede and Takagi [2008]. The two notions are closely related, but different. Their relationship is similar to how dlt singularities compare to klt singularities. Here we will discuss the former notion which in the dlt versus klt analogy corresponds to dit.

In this subsection we work over an algebraically closed field $k$, although in the rest of the paper we restrict to working over the complex numbers.

Definition 2.1. Let $X$ be a normal variety and $D \subseteq X$ an integral Weil divisor on $X$. A $\log$ resolution $\left(Y, D_{Y}\right) \stackrel{\pi}{\longrightarrow}(X, D)$ is a resolution of singularities such that $D_{Y}$ is the strict transform of $D$, and such that $\left(D_{Y}\right)_{\text {red }} \cup \operatorname{exc}(\pi)$ is a simple normal crossing divisor.

Definition 2.2. A reduced pair $(X, D)$ consists of a normal variety $X$ and a reduced divisor $D$ on $X$. For the definition of an snc pair, the strata of an snc pair and other normal crossing conditions please refer to [Kollár 2013, Definition 1.7].

One frequently wants log resolutions that do not blow up unnecessary centers. One good way to achieve this is with a thrifty resolution.

Definition 2.3 (thrifty resolution [Kollár 2013, Definition 2.79]). Let $(X, D)$ be a reduced pair. A thrifty resolution of $(X, D)$ is a resolution $\pi: Y \rightarrow X$ such that:

(a) $D_{Y}=\pi_{*}^{-1} D$ is a simple normal crossing divisor.

(b) $\pi$ is an isomorphism over the generic point of every stratum of the snc locus of $(X, D)$ and $\pi$ is an isomorphism at the generic point of every stratum of $\left(Y, D_{Y}\right)$.

Item (b) can also be replaced by:

$\left(\mathrm{b}^{\prime}\right)$ The exceptional set $E$ of $\pi$ does not contain any stratum of $\left(Y, D_{Y}\right)$ and $\pi(E)$ does not contain any stratum of the simple normal crossing locus of $(X, D)$.

We can now define rational pairs [Kollár 2013, Section 2.5].

Definition 2.4 (rational pairs). A reduced pair $(X, D)$ is called a rational pair if there exists a thrifty resolution $\pi:\left(Y, D_{Y}\right) \rightarrow(X, D)$ such that:

(i) $\mathscr{O}_{X}(-D) \simeq \pi_{*} \mathscr{O}_{Y}\left(-D_{Y}\right)$.

(ii) $\mathcal{R}^{i} \pi_{*} \mathscr{O}_{Y}\left(-D_{Y}\right)=0$ for all $i>0$.

(iii) $\mathcal{R}^{i} \pi_{*} \omega_{Y}\left(D_{Y}\right)=0$ for all $i>0$. 
If $(X, D)$ is a rational pair, and is in characteristic zero, then every thrifty resolution satisfies the properties (i), (ii), (iii) above [Kollár 2013, Corollary 2.86]. Even better though, property (iii) always holds in characteristic zero, as we point out below, whether or not $(X, D)$ is a rational pair.

Theorem 2.5. Assume that char $k=0$, and let $(X, D)$ be a reduced pair and $\pi:\left(Y, D_{Y}\right) \rightarrow(X, D)$ a thrifty resolution. Then $\mathcal{R}^{i} \pi_{*} \omega_{Y}\left(D_{Y}\right)=0$ for all $i>0$.

Proof. This follows from [Kollár 2013, Theorem 10.39].

Alternatively, one can prove Theorem 2.5 directly:

Claim 2.6. Let $\pi: E \rightarrow D$ be a proper birational map between reduced equidimensional $\mathbb{C}$-schemes of finite type such that $E$ is a simple normal crossing divisor in some smooth ambient space. Assume that $\pi$ is birational onto its image when restricted to every strata of $E$ (in particular, also each irreducible component of $E$ ). Then $\mathcal{R}^{i} \pi_{*} \omega_{E}=0$ for $i>0$.

Proof. We proceed by induction on $\operatorname{dim} D$ and the number of irreducible components of $E$, and note that the base case is simply Grauert-Riemenschneider vanishing [Grauert and Riemenschneider 1970]. Write $E=E_{0} \cup E^{\prime}$, where $E_{0}$ is an irreducible component of $E$ and $E^{\prime}$ denotes the remaining irreducible components. We have a short exact sequence

$$
0 \rightarrow \mathscr{O}_{E} \rightarrow \mathscr{O}_{E_{0}} \oplus \mathscr{O}_{E^{\prime}} \rightarrow \mathscr{O}_{E_{0} \cap E^{\prime}} \rightarrow 0
$$

Dualizing we obtain

$$
0 \rightarrow \omega_{E_{0}} \oplus \omega_{E^{\prime}} \rightarrow \omega_{E} \rightarrow \omega_{E_{0} \cap E^{\prime}} \rightarrow 0 .
$$

The intersection $E_{0} \cap E^{\prime}$ is a simple normal crossing divisor in the smooth ambient space $E_{0}$. It is also a union of strata of $E$ and hence $\pi$ is still birational when restricted to each strata of $E^{\prime} \cap E_{0}$. Applying $\mathcal{R}^{i} \pi_{*}$ and the inductive hypothesis to $E_{0}, E^{\prime}$ and $E_{0} \cap E^{\prime}$ proves the claim.

Alternative proof of Theorem 2.5. Push forward the short exact sequence

$$
0 \rightarrow \omega_{Y} \rightarrow \omega_{Y}\left(D_{Y}\right) \rightarrow \omega_{D_{Y}} \rightarrow 0
$$

via $\pi$ and apply the claim to $\omega_{D_{Y}}$ and $\omega_{Y}$. Note that the thrifty resolution hypothesis guarantees that $\pi$ is birational when restricted to any strata of $D_{Y}$ (since it is an isomorphism at the generic point of each strata).

This gives us the following criterion.

Proposition 2.7. Let $(X, D)$ be a reduced pair and $\pi:\left(Y, D_{Y}\right) \rightarrow(X, D)$ a thrifty resolution. Then $(X, D)$ is a rational pair if and only if

$$
\mathcal{R} \mathcal{H o m}_{\mathscr{O}_{X}}\left(\mathscr{O}_{X}(-D), \omega_{X}^{\cdot}\right) \simeq \mathcal{R} \pi_{*} \omega_{Y}\left(D_{Y}\right)[\operatorname{dim} X] \simeq \pi_{*} \omega_{Y}\left(D_{Y}\right)[\operatorname{dim} X]
$$


for some thrifty resolution. Furthermore, in characteristic zero the second isomorphism is automatic.

Proof. Observe that the conditions (i) and (ii) of Definition 2.4 are equivalent to the isomorphism $\mathcal{R} \pi_{*} \mathscr{O}_{Y}\left(-D_{Y}\right) \simeq \mathscr{O}_{X}(-D)$. Applying Grothendieck duality and condition (iii) to this isomorphism yields the statement. The characteristic zero statement is simply Theorem 2.5.

2B. Notation. Throughout the rest of this paper, all schemes will be assumed to be Noetherian separated schemes and essentially ${ }^{1}$ of finite type over $\mathbb{C}$. Given divisors $D=\sum a_{i} D_{i}$ and $D^{\prime}=\sum b_{i} D_{i}$ on a normal variety (possibly allowing $a_{i}, b_{j}$ to be zero), we define

$$
D \vee D^{\prime}=\sum \max \left(a_{i}, b_{i}\right) D_{i} \quad \text { and } \quad D \wedge D^{\prime}=\sum \min \left(a_{i}, b_{i}\right) D_{i} .
$$

Of course, if $D$ and $D^{\prime}$ have no common components then $D \vee D^{\prime}=D+D^{\prime}$ and $D \wedge D^{\prime}=0$. On a scheme $X$ essentially of finite type over $\mathbb{C}$, we use $\boldsymbol{D}\left({ }_{-}\right)=$ $\mathcal{R} \mathcal{H o m}_{\mathscr{C}_{X}}\left({ }_{-}, \omega_{X}^{*}\right)$ to denote the Grothendieck duality functor.

2C. Du Bois pairs. The notion of Du Bois singularities is becoming more and more part of basic knowledge in higher-dimensional geometry. In particular, for the notion of the Deligne-Du Bois complex of a scheme of finite type over $\mathbb{C}$ and its degree zero associated graded complex, denoted by $\underline{\Omega}_{X}^{0}$, we refer the reader to [Kollár 2013, Section 6.1].

In contrast, the notion of Du Bois pairs is relatively new and so here we discuss some of its basic properties.

Given a (possibly nonreduced) subscheme $Z \subseteq X$ one has an induced map in $D_{\mathrm{coh}}^{b}(X)$,

$$
\underline{\Omega}_{X}^{0} \rightarrow \underline{\Omega}_{Z}^{0}
$$

noting that by definition $\underline{\Omega}_{Z}^{0}=\underline{\Omega}_{Z_{\text {red }}}^{0}$. Then $\underline{\Omega}_{X, Z}^{0}$ is defined to be the object in the derived category making the following an exact triangle:

$$
\underline{\Omega}_{X, Z}^{0} \rightarrow \underline{\Omega}_{X}^{0} \rightarrow \underline{\Omega}_{Z}^{0} \stackrel{+1}{\rightarrow} .
$$

If $\mathscr{I}_{Z}$ is the ideal sheaf of $Z$, then it is easy to see that there is a natural map $\mathscr{I}_{Z} \rightarrow \underline{\Omega}_{X, Z}^{0}$ [Kovács 2011a, Section 3.D].

Definition 2.8 [Kovács 2011a, Definition 3.13]. We say that $(X, Z)$ is a $D u$ Bois pair (or simply a DB pair) if the above map $\mathscr{I}_{Z} \rightarrow \underline{\Omega}_{X, Z}^{0}$ is a quasi-isomorphism.

In the original definition of a Du Bois pair in [Kovács 2011a] it was assumed that $Z$ is reduced. As it turns out, this is not a necessary hypothesis.

$1_{\text {that is, a localization of a finite type scheme }}$ 
Lemma 2.9. If $(X, Z)$ is a Du Bois pair and $X$ is reduced, then $Z$ is reduced.

Proof. Note that $\underline{\Omega}_{Z}^{0}=\underline{\Omega}_{Z_{\text {red }}}^{0}$ and so $\underline{\Omega}_{X, Z}^{0} \simeq \underline{\Omega}_{X, Z_{\text {red }}}^{0}$. On the other hand, we also have an exact sequence,

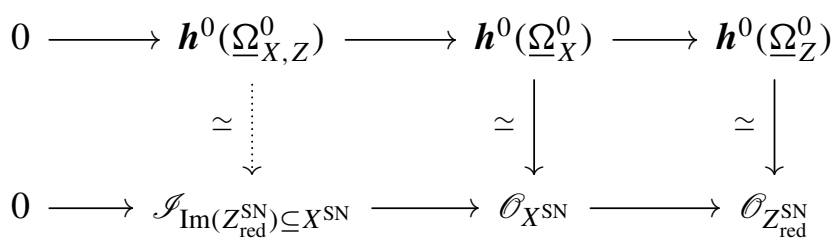

where $X^{\mathrm{SN}}, Z_{\text {red }}^{\mathrm{SN}}$ are the seminormalizations of $X$ and $Z_{\text {red }}$ respectively, and the right two isomorphisms come from [Saito 2000]. Note that the scheme-theoretic image of $Z_{\text {red }}^{\mathrm{SN}}$ in $X^{\mathrm{SN}}$ is reduced. The fact that the left-most vertical map is an isomorphism implies that $\boldsymbol{h}^{0}\left(\underline{\Omega}_{X, Z}^{0}\right)$ is a radical ideal in $\mathscr{O}_{X} \mathrm{SN}$. Since $(X, Z)$ is Du Bois, we see that $\boldsymbol{h}^{0}\left(\underline{\Omega}_{X, Z}^{0}\right)=\mathscr{I}_{Z \subseteq X}$ and hence $\mathscr{I}_{Z \subseteq X}$ is radical in $\mathscr{O}_{X}$ SN and hence also in $\mathscr{O}_{X}=\mathscr{O}_{X}$ red as desired.

Frequently we will take the Grothendieck dual of $\underline{\Omega}_{X, Z}^{0}$. Hence, following the notation of [Kovács and Schwede 2011a], we will write

$$
\underline{\omega}_{X, Z}^{\bullet}:=\mathcal{R} \mathcal{H o m}_{\mathscr{O}_{X}}\left(\underline{\Omega}_{X, Z}^{0}, \omega_{X}^{\bullet}\right)
$$

The reader is referred to [Kollár 2013, Section 6.1] for basic properties of Du Bois pairs. As mentioned in the introduction, this notion of pairs is somewhat different in flavor from the definition of $(X, Z)$ being log canonical or log terminal. Being a Du Bois pair is more a statement about the relationship between $X$ and $Z$, not an absolute statement about the singularities of $X$ or $Z$ separately. In particular, Examples 2.10 and 2.14 show that $(X, Z)$ being Du Bois does not imply that $X$ is Du Bois.

Example 2.10 (Du Bois pair whose ambient space is not Du Bois). Let $R$ denote the pullback of the diagram

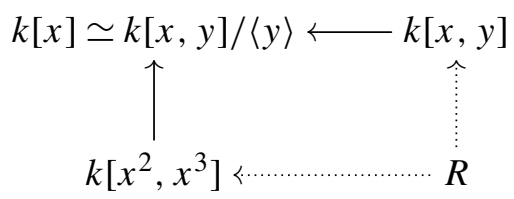

where the nondotted arrows are induced in the obvious ways. It is easy to see that $R=k\left[x^{2}, x^{3}, y, y x\right]$. By construction $X=\operatorname{Spec} R$ is not Du Bois since it is not seminormal. However, we claim that the pair $\left(\operatorname{Spec} R, V\left(\langle y, y x\rangle_{R}\right)\right)$ is Du Bois. 
Consider the following diagram:

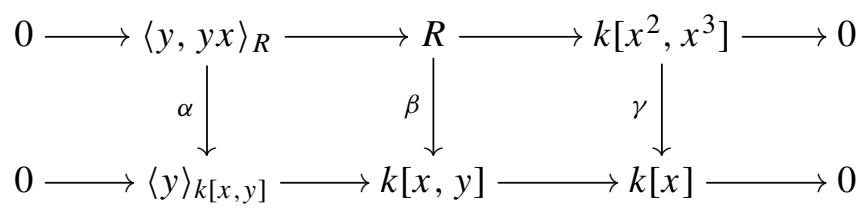

The maps labeled $\beta$ and $\gamma$ are the seminormalizations but $\alpha$ is an isomorphism. On the other hand, we know that $\underline{\Omega}_{X}^{0}=\underline{\Omega}_{X}^{0}$ in in general since they have the same hyperresolution. Therefore, up to harmless identification of modules with sheaves on an affine scheme, we see $\underline{\Omega}_{\mathrm{Spec} R}^{0} \simeq k[x, y]$ and $\underline{\Omega}_{\mathrm{Spec} k\left[x^{2}, x^{3}\right]}^{0} \simeq k[x]$ and so

$$
\langle y, y x\rangle_{R}=\langle y\rangle_{k[x, y]} \simeq \underline{\Omega}_{\operatorname{Spec~} R, V\left(\langle y, y x\rangle_{R}\right)}^{0} .
$$

This proves that ( $\left.\operatorname{Spec} R, V\left(\langle y, y x\rangle_{R}\right)\right)$ is Du Bois and completes the example.

Next we will give an example of a normal Du Bois pair whose ambient space is not Du Bois. To this end we will use a criterion for a cone being a Du Bois pair. In order to do that we need to recall a definition [Kollár 2013, III.3.8].

Let $X$ be a projective scheme and $\mathscr{L}$ an ample line bundle on $X$. We will need the spectrum of the section ring of $\mathscr{L}$,

$$
C_{a}(X, \mathscr{L}):=\operatorname{Spec}_{k} \bigoplus_{p \geq 0} H^{0}\left(X, \mathscr{L}^{p}\right)
$$

which is also called the (generalized) ample cone over $X$ with conormal bundle $\mathscr{L}$. If no confusion is likely, in particular when $\mathscr{L}$ is fixed, we will use the shorthand of $C X:=C_{a}(X, \mathscr{L})$. Notice that for a subscheme $Z \subseteq X$ there is a natural map $\iota: C_{a}\left(Z,\left.\mathscr{L}\right|_{Z}\right) \rightarrow C_{a}(X, \mathscr{L})$ which is a closed embedding away from the vertex $P \in C X$. By a slight abuse of notation we will also use $(C X, C Z)$ to denote the pair $\left(C_{a}(X, \mathscr{L}), \iota\left(C_{a}\left(Z,\left.\mathscr{L}\right|_{Z}\right)\right)\right)$.

Now we are ready to state the needed Du Bois criterion.

Proposition 2.11 ([Graf and Kovács 2014], cf. [Ma 2015]). Let X be a smooth projective variety, $Z \subset X$ an snc divisor (possibly the empty set), and $\mathscr{L}$ an ample line bundle on $X$. Then $(C X, C Z)$ is a Du Bois pair if and only if

$$
H^{i}\left(X, \mathscr{L}^{m}(-Z)\right)=0
$$

for all $i, m>0$.

Proof. If $Z=\varnothing$, this follows from [Ma 2015, Theorem 4.4]. The general case works similarly. For a direct proof see [Graf and Kovács 2014, Theorem 2.5].

While the above is sufficient for our purposes, we also obtained independently a slightly different statement using similar methods. 
Lemma 2.12 (Du Bois pairs for graded rings). Let $X$ be a projective variety with $D u$ Bois singularities, $\mathscr{L}$ an ample line bundle and $D$ a reduced connected divisor on $X$. Assume that $D$ also has only Du Bois singularities. Form the corresponding section ring $S=\bigoplus_{i \geq 0} \Gamma\left(X, \mathscr{L}^{i}\right)$ and $I=\bigoplus_{i \geq 0} \Gamma\left(X, \mathscr{O}_{X}(-D) \otimes \mathscr{L}^{i}\right)$. Fix $\mathfrak{m}=S_{+}$ to be the irrelevant ideal. Set $Y=C X=\operatorname{Spec} S$ and $Z=C D=\operatorname{Spec}(S / I)$. If

$$
H^{1}\left(X, \mathscr{O}_{X}(-D) \otimes \mathscr{L}^{i}\right)=0
$$

for $i \geq 0$ so that $S / I \simeq \bigoplus_{i \geq 0} \Gamma\left(D,\left.\mathscr{L}^{i}\right|_{D}\right)$, then for all $i \geq 1$ we have

$$
\boldsymbol{h}^{i}\left(\underline{\Omega}_{Y, Z}^{0}\right) \simeq\left[H_{\mathfrak{m}}^{i+1}(I)\right]_{>0} .
$$

Again under hypothesis (2.12.1), we see immediately that $(Y, Z)$ is Du Bois if and only if $\left[H_{\mathfrak{m}}^{i}(I)\right]_{>0}=0$ for every $i>0$.

Proof. First observe that both $Y$ and $Z$ are seminormal since they are saturated section rings over seminormal schemes. L. Ma [2015, Equation (4.4.4) in the proof of Theorem 4.4] showed that

$$
\boldsymbol{h}^{i}\left(\underline{\Omega}_{Y}^{0}\right) \simeq\left[H_{\mathfrak{m}}^{i+1}(S)\right]_{>0}
$$

for $i>0$. Likewise $\boldsymbol{h}^{i}\left(\underline{\Omega}_{Z}^{0}\right)=\left[H_{\mathfrak{m}}^{i+1}(S / I)\right]_{>0}$ for $i>0$. Now we analyze $\boldsymbol{h}^{i+j}\left(\mathcal{R} \Gamma_{\mathfrak{m}}\left(\underline{\Omega}_{Y}^{0}\right)\right)$ via a spectral sequence. Since $Y$ is Du Bois outside of the origin $V(\mathfrak{m})$, we see that $\boldsymbol{h}^{j}\left(\underline{\Omega}_{Y}^{0}\right)$ is supported only at the origin for $j>0$. It follows that the $E_{2}$-page of the spectral sequence

$$
H_{\mathfrak{m}}^{i}\left(\boldsymbol{h}^{j}\left(\underline{\Omega}_{Y}^{0}\right)\right) \Rightarrow \boldsymbol{h}^{i+j}\left(\mathcal{R} \Gamma_{\mathfrak{m}}\left(\underline{\Omega}_{Y}^{0}\right)\right)
$$

looks like

$\begin{array}{cccccc}\boldsymbol{h}^{3}\left(\underline{\Omega}_{Y}^{0}\right) & 0 & 0 & 0 & 0 & \cdots \\ \boldsymbol{h}^{2}\left(\underline{\Omega}_{Y}^{0}\right) & 0 & 0 & 0 & 0 & \cdots \\ \boldsymbol{h}^{1}\left(\underline{\Omega}_{Y}^{0}\right) \underbrace{H_{\mathfrak{m}}^{1}(S)}_{0}{ }^{H_{\mathfrak{m}}^{2}(S)} & H_{\mathfrak{m}}^{3}(S) & H_{\mathfrak{m}}^{4}(S) & \cdots\end{array}$

Here we are using the fact that $S$ is seminormal, and so $\boldsymbol{h}^{0}\left(\underline{\Omega}_{Y}^{0}\right)=S$. It is not difficult to see that the unique nonzero map of the $(i-1)$-st page of this spectral sequence induces the isomorphism of (2.12.2), and so those unique nonzero maps 
are injective. Thus the spectral sequence contains the data of a long exact sequence $0 \rightarrow H_{\mathfrak{m}}^{1}(S) \rightarrow \mathbb{H}_{\mathfrak{m}}^{1}\left(\underline{\Omega}_{Y}^{0}\right) \rightarrow \boldsymbol{h}^{1}\left(\underline{\Omega}_{Y}^{0}\right) \hookrightarrow H_{\mathfrak{m}}^{2}(S) \rightarrow \mathbb{H}_{\mathfrak{m}}^{2}\left(\underline{\Omega}_{Y}^{0}\right) \rightarrow \boldsymbol{h}^{2}\left(\underline{\Omega}_{Y}^{0}\right) \hookrightarrow H_{\mathfrak{m}}^{3}(S) \rightarrow \cdots$.

Hence $\mathbb{\boxplus}_{\mathfrak{m}}^{i}\left(\underline{\Omega}_{Y}^{0}\right)=\left[H_{\mathfrak{m}}^{i}(S)\right]_{\leq 0}$ for $i \geq 2$ and $\mathbb{\boxplus}_{\mathfrak{m}}^{1}\left(\underline{\Omega}_{Y}^{0}\right) \simeq H_{\mathfrak{m}}^{1}(S)$. Likewise $\mathbb{H}_{\mathfrak{m}}^{i}\left(\underline{\Omega}_{Z}^{0}\right)=$ $\left[H_{\mathfrak{m}}^{i}(S / I)\right]_{\leq 0}$ for $i \geq 2$ and $\mathbb{W}_{\mathfrak{m}}^{1}\left(\Omega_{Z}^{0}\right) \simeq H_{\mathfrak{m}}^{1}(S / I)$. Furthermore, since $Y$ and $Z$ are seminormal we see that $\boldsymbol{h}^{0}\left(\underline{\Omega}_{X, Z}^{0}\right)=I$ and so the same spectral sequence argument implies that we have a long exact sequence

$0 \rightarrow H_{\mathfrak{m}}^{1}(I) \rightarrow \mathbb{H}_{\mathfrak{m}}^{1}\left(\underline{\Omega}_{Y, Z}^{0}\right) \rightarrow \boldsymbol{h}^{1}\left(\underline{\Omega}_{Y, Z}^{0}\right) \rightarrow H_{\mathfrak{m}}^{2}(I) \rightarrow \mathbb{U}_{\mathfrak{m}}^{2}\left(\underline{\Omega}_{Y, Z}^{0}\right) \rightarrow \boldsymbol{h}^{2}\left(\underline{\Omega}_{Y, Z}^{0}\right) \rightarrow H_{\mathfrak{m}}^{3}(I) \rightarrow \cdots$.

We still have the labeled surjectivities by the Matlis dual of Theorem 3.2, which we will prove later (we assume it for now). Thus it is enough to see that the maps above make the identification $\mathbb{H}_{\mathfrak{m}}^{i}\left(\underline{\Omega}_{Y, Z}^{0}\right)=\left[H_{\mathfrak{m}}^{i}(I)\right]_{\leq 0}$ for $i \geq 2$.

We consider the diagram with distinguished triangles as rows

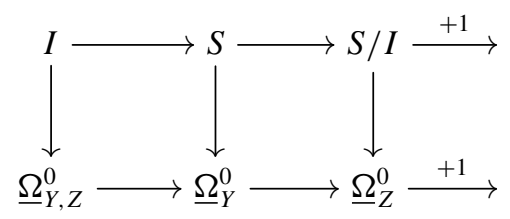

We will apply the functor $\mathcal{R} \Gamma_{\mathfrak{m}}\left({ }_{-}\right)$and take cohomology $i \geq 1$ to obtain

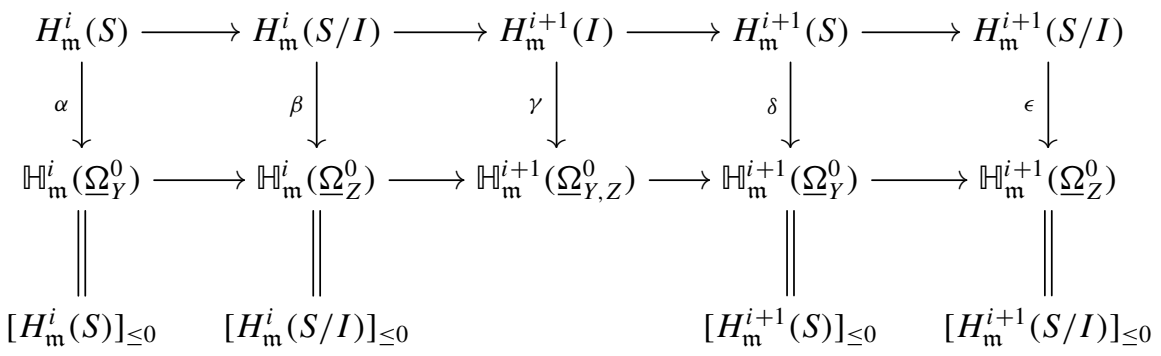

Note that $\gamma$ is the map we already identified as surjective above. It is easy to see that the vertical maps $\alpha, \beta, \delta$, and $\epsilon$ are the projections and so $[\alpha]_{\leq 0},[\beta]_{\leq 0},[\delta]_{\leq 0}$, and $[\epsilon]_{\leq 0}$ are isomorphisms. Thus $[\gamma]_{\leq 0}$ is also an isomorphism. But from the second row we see that $\mathbb{W}_{\mathfrak{m}}^{i}\left(\underline{\Omega}_{Y, Z}^{0}\right)$ is of nonpositive degree so that $\mathbb{W}_{\mathfrak{m}}^{i}\left(\underline{\Omega}_{Y, Z}^{0}\right)=\left[H_{\mathfrak{m}}^{i}(I)\right]_{\leq 0}$ for $i \geq 2$.

Remark 2.13. It would be natural to try to prove a common generalization of the (independently obtained) Proposition 2.11 and Lemma 2.12.

Example 2.14 (a normal Du Bois pair whose ambient space is not Du Bois). Let $W$ be an arbitrary smooth canonically polarized variety, that is, $W$ is smooth and projective and $\omega_{W}$ is ample. Further let $n>1$, and set $X=W \times \mathbb{P}^{n}$ and $\mathscr{L}=\pi_{1}^{*} \omega_{W} \otimes \pi_{2}^{*} \mathscr{O}_{\mathbb{P}^{n}}(1)$. Finally, let $K \subseteq \mathbb{P}^{n}$ be a smooth hypersurface of degree 
$n+1$, that is, $\mathscr{O}_{\mathbb{P}^{n}}(K) \simeq \omega_{\mathbb{P}^{n}}^{-1}$, and let $Z=W \times K$. We claim that, using the above notation, $(C X, C Z)$ is a Du Bois pair, while $C X$ itself is not. Note also that by construction $C X$ is normal.

Consider $H^{1}\left(X, \mathscr{O}_{X}(-Z) \otimes \mathscr{L}^{j}\right)$ for $j \geq 0$ and observe that $\mathscr{O}_{X}(-Z) \otimes \mathscr{L}^{j}=\pi_{2}^{*} \mathscr{O}_{\mathbb{P}^{n}}(-n-1) \otimes \pi_{1}^{*} \omega_{W}^{j} \otimes \pi_{2}^{*} \mathscr{O}_{\mathbb{P}^{n}}(j)=\pi_{1}^{*} \omega_{W}^{j} \otimes \pi_{2}^{*} \mathscr{O}_{\mathbb{P}^{n}}(j-n-1)$.

Now $H^{1}\left(\mathbb{P}^{n}, \mathscr{O}_{\mathbb{P}^{n}}(j-n-1)\right)=0$ for all $j \geq 0$ and $H^{0}\left(\mathbb{P}^{n}, \mathscr{O}_{\mathbb{P}^{n}}(j-n-1)\right)=0$ for $j \leq n$. But if $j>n \geq 1$, then $H^{1}\left(W, \omega_{W}^{j}\right)=0$ by Kodaira vanishing and so it follows by the Künneth formula that $H^{1}\left(X, \mathscr{O}_{X}(-Z) \otimes \mathscr{L}^{j}\right)=0$ for all $j \geq 0$, so the hypotheses of Lemma 2.12 are satisfied.

Let $r=\operatorname{dim} W$ and consider $H^{r}(X, \mathscr{L})$. By the Künneth formula

$$
H^{r}(X, \mathscr{L}) \supseteq H^{r}\left(W, \omega_{W}\right) \otimes H^{0}\left(\mathbb{P}^{n}, \mathscr{O}_{\mathbb{P}^{n}}(1)\right) \neq 0,
$$

and hence by Proposition 2.11 CX is not Du Bois.

On the other hand we have that

$$
\mathscr{L}(-Z) \simeq \pi_{1}^{*} \omega_{W} \otimes \pi_{2}^{*} \mathscr{O}_{\mathbb{P}^{n}}(1-n-1) \simeq \omega_{X} \otimes \pi_{2}^{*} \mathscr{O}_{\mathbb{P}^{n}}(1) .
$$

Now observe that $H^{q}\left(\mathbb{P}^{n}, \mathscr{O}_{\mathbb{P}^{n}}(1-n-1)\right)=0$ for all $q \geq 0$, so, again by the Künneth formula, it follows that $H^{i}(X, \mathscr{L}(-Z))=0$ for all $i>0$.

In order to conclude that $(C X, C Z)$ is a Du Bois pair we need that

$$
H^{i}\left(X, \mathscr{L}^{m}(-Z)\right)=0 \text { for all } i, m>0 .
$$

We just showed that $H^{i}(X, \mathscr{L}(-Z))=0$ for all $i>0$, which handles the $m=1$ case. If $m>1$ then $\mathscr{M}:=\mathscr{L}^{m-1} \otimes \pi_{2}^{*} \mathscr{O}_{\mathbb{P}^{n}}(1)$ is ample on $X$ and by (2.14.1) and Kodaira vanishing we have that

$$
H^{i}\left(X, \mathscr{L}^{m}(-Z)\right)=H^{i}\left(X, \mathscr{L}(-Z) \otimes \mathscr{L}^{m-1}\right) \simeq H^{i}\left(X, \omega_{X} \otimes \mathscr{M}\right)=0,
$$

and hence it follows from Proposition 2.11 that $(C X, C Z)$ is indeed a Du Bois pair.

We will find the following lemma useful; cf. [Esnault 1990; Schwede 2007].

Lemma 2.15. Assume that $(X, Z)$ is a pair with $Z \subseteq X$ reduced schemes. Assume further that $X \subseteq Y$ where $Y$ is smooth. Let $\pi: \widetilde{\widetilde{Y}} \rightarrow Y$ be a log resolution of both $X$ and $Z$ in $Y$ and set $\bar{X}$ and $\bar{Z}$ to be the reduced preimages of $X$ and $Z$ in $\tilde{Y}$ respectively. Then

$$
\underline{\Omega}_{X, Z}^{0} \simeq \mathcal{R} \pi_{*} \mathscr{I}_{\bar{Z} \subseteq \bar{X}}
$$

where $\mathscr{I}_{\bar{Z} \subseteq \bar{X}}$ is the ideal of $\bar{Z}$ in $\bar{X}$. 
Proof. Consider the diagram

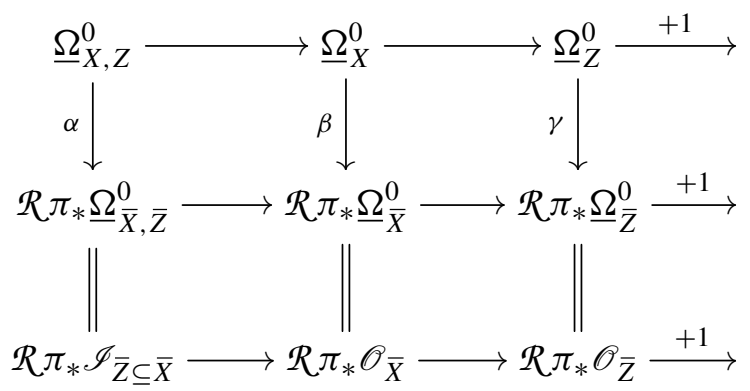

The vertical arrows $\beta$ and $\gamma$ are quasi-isomorphisms by [Kovács and Schwede 2011b, Theorem 6.4] (also see [Schwede 2007, Theorem 4.3]) since $\bar{X}$ and $\bar{Z}$ are snc and hence Du Bois. The second row of equalities also follows since $\bar{X}$ and $\bar{Z}$ are $\mathrm{Du}$ Bois. Then $\alpha$ is a quasi-isomorphism as well and hence the lemma follows.

There are some situations when a pair being Du Bois implies that the ambient space is also Du Bois. It is proved in [Graf and Kovács 2014] that this happens if $X$ is Gorenstein, but that $X$ being $\mathbb{Q}$-Gorenstein is not sufficient. Another simple situation in which this holds is the following.

Lemma 2.16. Let $X$ be a reduced $\mathbb{C}$-scheme essentially of finite type and $H$ a Cartier divisor. If $(X, H)$ is a Du Bois pair then $X$ (and hence $H$ ) is also Du Bois.

Proof. The statement is local and so we may assume that $X=\operatorname{Spec} R$ is affine. We know that $\mathscr{O}_{X}(-H) \rightarrow \underline{\Omega}_{X, H}^{0}$ is a quasi-isomorphism and thus so is $\mathscr{O}_{X} \rightarrow$ $\underline{\Omega}_{X, H}^{0} \otimes \mathscr{O}_{X}(H)$. We will show that this map factors through $\mathscr{O}_{X} \rightarrow \underline{\Omega}_{X}^{0}$, which will complete the proof by [Kovács 1999, Theorem 2.3].

Embed $X \subseteq Y$ as a smooth scheme and let $\pi: \widetilde{Y} \rightarrow Y$ be a simultaneous log resolution of $(Y, X)$ and $(Y, H)$ with $\bar{X}, \bar{H}$ the reduced total transforms of $X$ and $H$ respectively. Then $\underline{\Omega}_{X, H}^{0}=\mathcal{R} \pi_{*} \mathscr{I}_{\bar{H} \subseteq \bar{X}}$ by Lemma 2.15 . Fix $X^{\prime}$ to be the components of $\bar{X}$ which are not also components of $\bar{H}$ and we see that $\mathscr{I}_{\bar{H} \subseteq \bar{X}} \simeq \mathscr{O}_{X^{\prime}}\left(-\left.\bar{H}\right|_{X^{\prime}}\right)$. Thus

$$
\underline{\Omega}_{X, H}^{0} \otimes \mathscr{O}_{X}(H) \simeq \mathcal{R} \pi_{*} \mathscr{O}_{X^{\prime}}\left(\left.\left(\pi^{*} H-\bar{H}\right)\right|_{X^{\prime}}\right) .
$$

Since $\pi^{*} H-\bar{H}$ is effective, we obtain a map

$$
\underline{\Omega}_{X}^{0} \simeq \mathcal{R} \pi_{*} \mathscr{O}_{\bar{X}} \rightarrow \mathcal{R} \pi_{*} \mathscr{O}_{X^{\prime}} \rightarrow \mathcal{R} \pi_{*} \mathscr{O}_{X^{\prime}}\left(\left.\left(\pi^{*} H-\bar{H}\right)\right|_{X^{\prime}}\right) \simeq \underline{\Omega}_{X, H}^{0} \otimes \mathscr{O}_{X}(H) .
$$

This map obviously factors the quasi-isomorphism $\mathscr{O}_{X} \rightarrow \underline{\Omega}_{X, H}^{0} \otimes \mathscr{O}_{X}(H)$ and hence the proof is complete.

We recall properties of $\underline{\Omega}_{X, Z}^{0}$ that we will need later.

Lemma 2.17. Let $X$ be a scheme over $\mathbb{C}$ with $Z \subseteq X$ a closed subscheme and $j: U=X \backslash Z \hookrightarrow X$ the complement of $Z$. Then: 
(a) If in addition $X$ is proper, then $H^{i}\left(X, \mathscr{I}_{Z}\right) \rightarrow \mathbb{H}^{i}\left(X, \Omega_{X, Z}^{0}\right)$ is surjective for all $i \in \mathbb{Z}$ [Kovács 2011a, Corollary 4.2; Kollár 2013, Theorem 6.22].

(b) If $H$ is a general member of a basepoint-free linear system, then $\underline{\Omega}_{X, Z}^{0} \otimes \mathscr{O}_{H} \simeq$ $\Omega_{H, H \cap Z}^{0}$ [Kovács 2011a, Proposition 3.18; Kollár 2013, Theorem 6.5(6)].

(c) If $X=U \cup V$ is a decomposition into closed subschemes and $Z \subseteq X$ is another closed subscheme, then we have a distinguished triangle

$$
\underline{\Omega}_{U \cup V, Z}^{0} \rightarrow \underline{\Omega}_{U, Z \cap U}^{0} \oplus \underline{\Omega}_{V, Z \cap V}^{0} \rightarrow \underline{\Omega}_{U \cap V, Z \cap U \cap V}^{0} \stackrel{+1}{\rightarrow}
$$

(cf. [Kollár 2013, Theorem 6.5(11)]).

(d) Let $X=U \cup V$ be a decomposition of $X$ into closed subschemes. Then

$$
\underline{\Omega}_{U \cup V, V}^{0} \simeq \underline{\Omega}_{U, U \cap V}^{0}
$$

(cf. [Kovács 2011a, Proposition 3.19; Kollár 2013, Theorem 6.17]).

Proof. Parts (a) and (b) follow from the references in their statements. For (c), the included reference only states the triangle in the case that $Z=\varnothing$. However, our more general version follows easily from the diagram

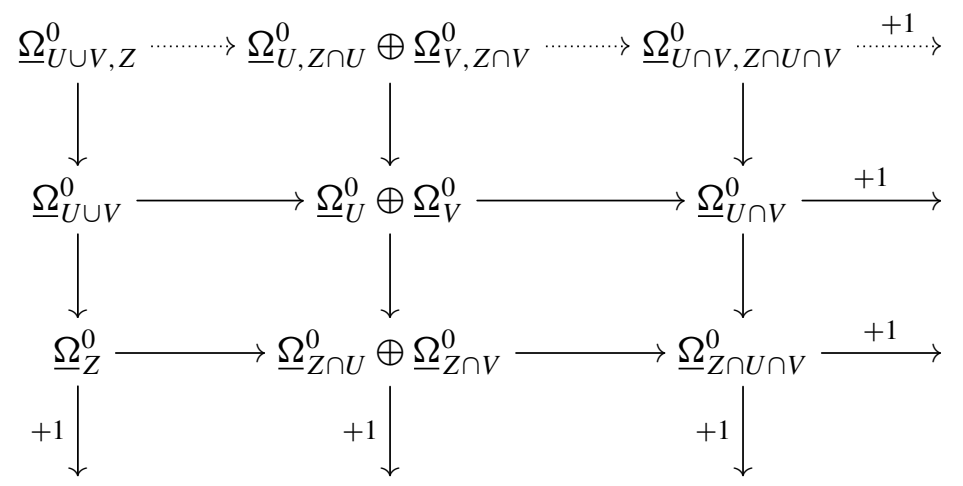

and the 9-lemma in triangulated categories [Kovács 2013, B.1].

For (d), consider the distinguished triangle

$$
\underline{\Omega}_{U \cup V}^{0} \longrightarrow \underline{\Omega}_{U}^{0} \oplus \underline{\Omega}_{V}^{0} \longrightarrow \underline{\Omega}_{U \cap V}^{0} \stackrel{+1}{\longrightarrow}
$$

of part (c) with $Z=\varnothing$. Then [Kollár and Kovács 2010, Lemma 2.1] implies that the left vertical arrow of the following diagram is an isomorphism:

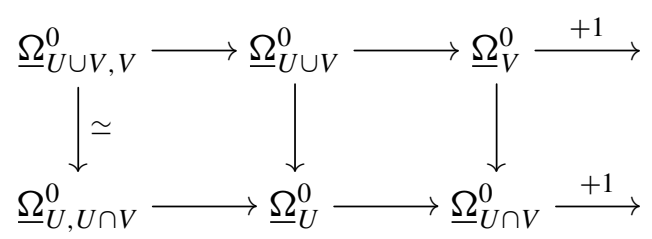


For more details see the proofs in the references and replace $\underline{\Omega}^{\times}$with $\underline{\Omega}^{0}$.

The next lemma constructs a natural exact triangle for Du Bois pairs.

Lemma 2.18. Let $X$ be a scheme and $W, Z \subseteq X$ subschemes. Then there is a distinguished triangle

$$
\underline{\Omega}_{X, W \cup Z}^{0} \rightarrow \underline{\Omega}_{X, Z}^{0} \rightarrow \underline{\Omega}_{W, Z \cap W}^{0} \stackrel{+1}{\longrightarrow} .
$$

In particular, because there is also a short exact sequence

$$
0 \rightarrow \mathscr{I}_{W \cup Z \subseteq X} \rightarrow \mathscr{I}_{Z \subseteq X} \rightarrow \mathscr{I}_{Z \cap W \subseteq W} \rightarrow 0
$$

if any two of $\{(X, W \cup Z),(X, Z),(W, Z \cap W)\}$ are Du Bois, so is the third.

Proof. We begin with a diagram of distinguished triangles as columns and rows (see [Kollár 2013, Theorem 6.5.11; Kovács 2013, Theorem B1]):

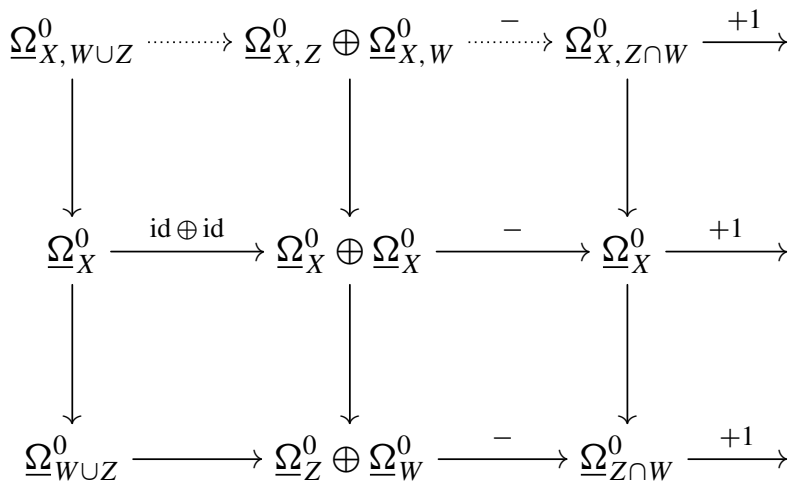

The horizontal maps in the second column of this diagram are each obtained by subtracting the canonical maps on each factor of the direct sum, hence the minus signs. The octahedral axiom implies that there exists a diagram of distinguished triangles,

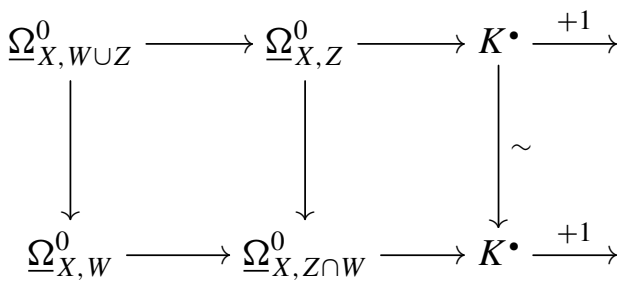

We need to identify $K^{\bullet}$. Notice that the bottom row also fits into another diagram of distinguished triangles (see [Kovács 2013, Theorem B1]): 


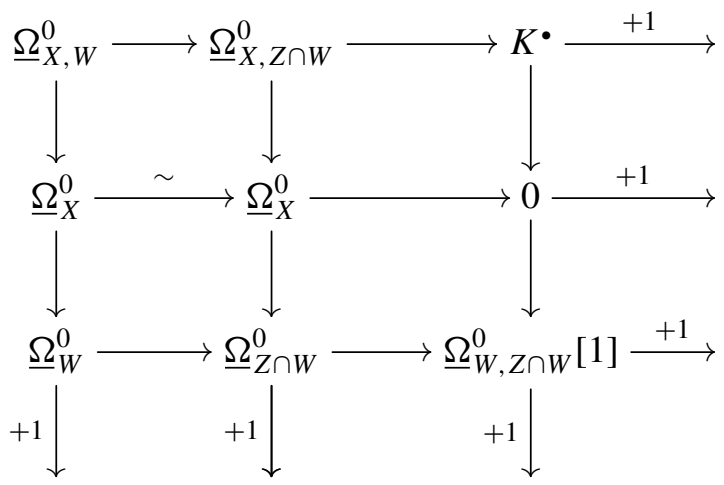

Hence $K^{\bullet} \simeq \underline{\Omega}_{W, Z \cap W}^{0}$ and the lemma follows.

Finally, note that being Du Bois is a direct generalization of being rational for pairs (see also Kollár 2013, Corollary 6.25).

Theorem 2.19 [Kovács 2011a, Corollary 5.6]. If $(X, D)$ is a rational pair then $(X, D)$ is also a Du Bois pair.

\section{An injectivity theorem}

A key ingredient of the proof that Du Bois singularities are deformation invariant was an injectivity theorem [Kovács and Schwede 2011a, Theorem 3.3]. In this section, we generalize that result to the context of pairs.

Lemma 3.1 (cf. [Kovács and Schwede 2011a, Lemma 3.1]). Let X be a reduced scheme, $Z \subseteq X$ a reduced subscheme and $\mathscr{L}$ a semiample line bundle. Let $s \in \mathscr{L}^{n}$ be a general global section for some $n \gg 0$ and take the $n$-th root of this section (as in [Kollár and Mori 1998, Definition 2.50]):

$$
\eta: Y=\operatorname{Spec} \bigoplus_{i=0}^{n-1} \mathscr{L}^{-i} \rightarrow X .
$$

Set $W=\eta^{-1}(Z)$ (with the induced scheme structure). Note that the restriction satisfies $\left.\eta\right|_{W}: W=$ Spec $\left.\bigoplus_{i=0}^{n-1} \mathscr{L}^{-i}\right|_{Z} \rightarrow Z$. Then as before, writing $\eta_{*}=\mathcal{R} \eta_{*}$,

$$
\eta_{*} \underline{\Omega}_{Y, W}^{0} \simeq \underline{\Omega}_{X, Z}^{0} \otimes \eta_{*} \mathscr{O}_{Y} \simeq \bigoplus_{i=0}^{n-1}\left(\underline{\Omega}_{X, Z}^{0} \otimes \mathscr{L}^{-i}\right),
$$

and this direct sum is compatible with the decomposition $\eta_{*} \mathscr{O}_{Y}=\bigoplus_{i=0}^{n-1} \mathscr{L}^{-i}$.

Proof. Although not explicitly stated, it is easy to see that [Kovács and Schwede 2011a, Lemma 3.1] is functorial in that it is compatible with the map $Z \rightarrow X$. Then 
by applying Lemma 2.17(b), the result follows from the diagram

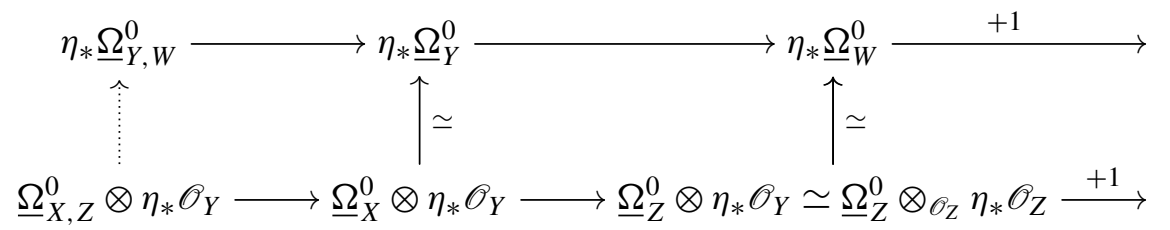

Setting $\underline{\omega}_{X, Z}^{\bullet}=\mathcal{R} \mathcal{H o m}_{\mathscr{O}_{X}}^{\bullet}\left(\underline{\Omega}_{X, Z}^{0}, \omega_{X}^{\bullet}\right)$ as in (2.9.1), we easily obtain the following.

Theorem 3.2. Let $X$ be a reduced scheme over $\mathbb{C}$ and $Z \subseteq X$ a reduced subscheme. Then the natural map

$$
\Phi^{j}: \boldsymbol{h}^{j}\left(\underline{\omega}_{X, Z}^{\bullet}\right) \hookrightarrow \boldsymbol{h}^{j}\left(\mathcal{R} \mathcal{H o m}_{\mathscr{O}_{X}}\left(\mathscr{I}_{Z}, \omega_{X}^{\bullet}\right)\right)
$$

is injective for every $j \in \mathbb{Z}$.

Proof. The proof is essentially the same as in [Kovács and Schwede 2011a, Theorem 3.3] so we only sketch it briefly. First, since the question is local and compatible with restricting to an open subset, we may assume that $X$ is projective with ample line bundle $\mathscr{L}$. It follows from taking a cyclic cover with respect to a general section of $\mathscr{L}^{n}$, for $n \gg 0$, and applying Lemmas 2.17(a) and 3.1 that

$$
H^{j}\left(X, \mathscr{I}_{Z} \otimes \bigoplus_{i=0}^{n-1} \mathscr{L}^{-i}\right) \rightarrow \mathbb{M}^{j}\left(X, \underline{\Omega}_{X, Z}^{0} \otimes \bigoplus_{i=0}^{n-1} \mathscr{L}^{-i}\right)
$$

surjects for all $j \geq 0$. Therefore $H^{j}\left(X, \mathscr{I}_{Z} \otimes \mathscr{L}^{-i}\right) \rightarrow \mathbb{M}^{j}\left(X, \underline{\Omega}_{X, Z}^{0} \otimes \mathscr{L}^{-i}\right)$ surjects for all $i, j \geq 0$.

By an application of Serre-Grothendieck duality we obtain an injection

$$
\mathbb{M}^{j}\left(X, \underline{\omega}_{X, Z}^{\cdot} \otimes \mathscr{L}^{i}\right) \hookrightarrow \mathbb{M}^{j}\left(X, \mathcal{R} \mathcal{H} m_{\mathscr{O}_{X}}^{\cdot}\left(\mathscr{I}_{Z}, \omega_{X}^{\bullet}\right) \otimes \mathscr{L}^{i}\right)
$$

for all $i, j \geq 0$. But for $i \gg 0$, by Serre vanishing, we obtain that

$$
H^{0}\left(X, \boldsymbol{h}^{j}\left(\underline{\omega}_{X, Z}^{\bullet}\right) \otimes \mathscr{L}^{i}\right) \hookrightarrow H^{0}\left(X, \boldsymbol{h}^{j}\left(\mathcal{R} \mathcal{H o m}_{\mathscr{O}_{X}}\left(\mathscr{I}_{Z}, \omega_{X}^{\bullet}\right)\right) \otimes \mathscr{L}^{i}\right)
$$

is injective as well (since the spectral sequence computing (3.2.1) degenerates). On the other hand, if $\boldsymbol{h}^{j}\left(\underline{\omega}_{X, Z}^{\cdot}\right) \rightarrow \boldsymbol{h}^{j}\left(\mathcal{R} \mathcal{H} m_{\mathscr{O}_{X}}\left(\mathscr{I}, \omega_{X}^{*}\right)\right)$ is not injective, then for some $i \gg 0$ neither is (3.2.2).

\section{Deformation of Du Bois pairs}

In [Kovács and Schwede 2011a, Corollary 4.2], we showed the following result: Let $f: X \rightarrow B$ be a flat proper family over a smooth curve $B$ with a fiber $X_{0}$, $0 \in B$, having $\mathrm{Du}$ Bois singularities. Then there is an open neighborhood $0 \in U \subseteq B$ such that the fibers $X_{u}$ have Du Bois singularities for $u \in U$. In this section, we 
generalize this result to Du Bois pairs. We mimic our previous approach as much as possible.

First we need a lemma, which is presumably well known but for which we know no reference.

Lemma 4.1. Let $X$ be a reduced scheme and $Z \subseteq X$ a reduced subscheme with ideal sheaf $\mathscr{I}_{Z}$. Further, let $H \subseteq X$ be an effective Cartier divisor with ideal sheaf $\mathscr{I}_{H}$ such that $H$ does not contain any irreducible components of either $X$ or $Z$. Then

$$
\mathscr{I}_{H} \cap \mathscr{I}_{Z}=\mathscr{I}_{H} \cdot \mathscr{I}_{Z}
$$

Proof. This is left as an exercise to the reader. Earlier versions of this paper, which are available on the arXiv, also contain a detailed proof.

Now we prove that if a special fiber supports a Du Bois pair, so does the total space near that fiber. Recall that effective Cartier divisors on a possibly nonnormal scheme are simply subschemes locally defined by a single non-zero-divisor near every point.

Theorem 4.2. Let $X$ be a reduced scheme essentially of finite type over $\mathbb{C}, Z \subseteq X$ a reduced subscheme and $H$ a reduced effective Cartier divisor on $X$ that does not contain any component of $Z$. If $(H, Z \cap H)$ is a Du Bois pair, then $(X, Z)$ is a Du Bois pair near $H$. It then follows (from Lemma 2.18) that $(X, Z \cup H)$ is Du Bois near H.

Proof. We follow very closely the proofs of [Kovács 2000, Theorem 3.2] and [Kovács and Schwede 2011a, Theorem 4.1], which are based on [Elkik 1978]. Choose a closed point $\mathfrak{q}$ of $X$ contained within $H$. It is sufficient to prove that $(X, Z)$ is Du Bois at $\mathfrak{q}$. Let $R$ denote the stalk $\mathscr{O}_{X, \mathfrak{q}}$ and replace $X$ by $\operatorname{Spec} R$. Choose $f \in R$ to denote a defining equation of $H$ in $R$. Consider the following diagram, whose rows are distinguished triangles in $D_{\mathrm{coh}}^{b}(X)$ :

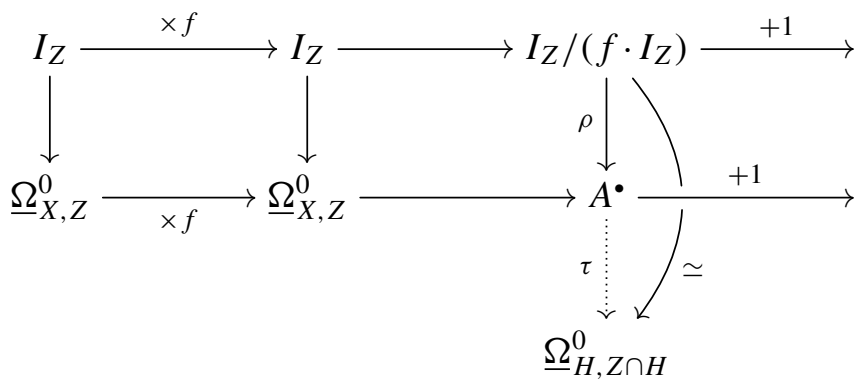

where $A^{\bullet}$ is the term completing the second row to a distinguished triangle. We claim we have a map $\tau$ as above such that $\tau \circ \rho$ is a quasi-isomorphism. Certainly 
we have a diagram with distinguished triangles for rows and columns

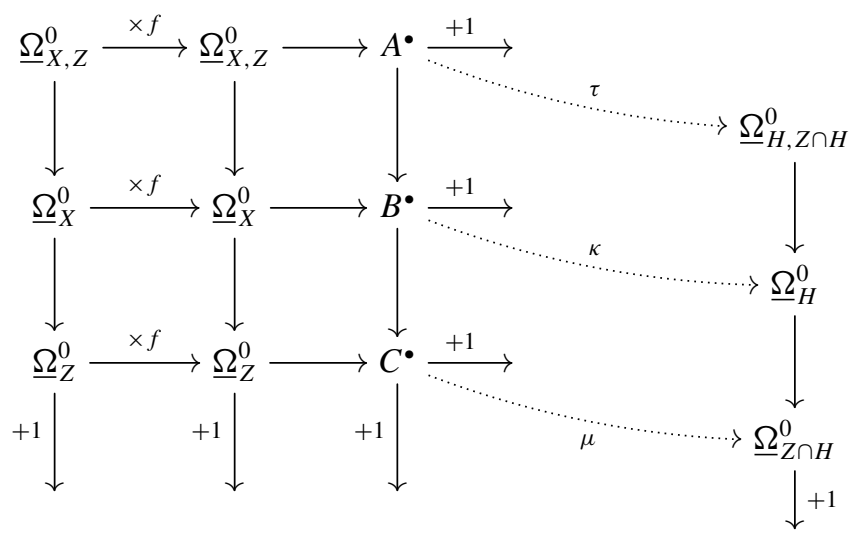

and the existence of $\tau$ follows immediately from the existence of $\kappa$ and $\mu$, whose existence follows from the proof of [Kovács and Schwede 2011a, Theorem 4.1]. Note that the assumptions imply that $\left.H\right|_{Z}=H \cap Z$ is a Cartier divisor on $Z$, so we may indeed use [Kovács and Schwede 2011a, Theorem 4.1] for both $X$ and $Z$. Since $I_{Z} /\left(f \cdot I_{Z}\right)=I_{Z} /\left((f) \cap I_{Z}\right)$ by Lemma 4.1 and because $(H, Z \cap H)$ is a Du Bois pair, we see $\tau \circ \rho$ is an isomorphism as claimed.

Next we apply the Grothendieck duality functor $\boldsymbol{D}\left({ }_{-}\right)=\mathcal{R} \operatorname{Hom}_{R}\left({ }_{-}, \omega_{R}^{*}\right)$ to (4.2.1) and take cohomology, using $\boldsymbol{k}^{i}\left({ }_{-}\right)$as shorthand to denote $\boldsymbol{h}^{i}\left(\boldsymbol{D}\left({ }_{-}\right)\right)$:

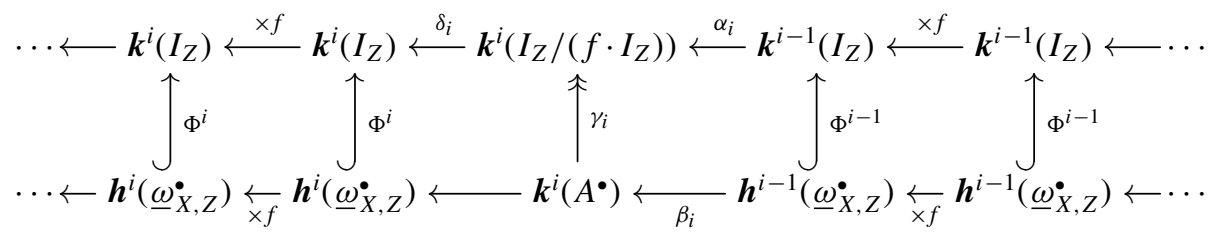

where the $\Phi^{\bullet}$ are injective by Theorem 3.2 and $\gamma_{i}$, which was obtained from $\rho$, is surjective since $\tau \circ \rho$ is an isomorphism.

The proof now follows exactly as for the main theorem of [Kovács and Schwede 2011a], or dually of [Kovács 2000, Theorem 3.2]. Fix $z \in \boldsymbol{h}^{i-1}\left(\boldsymbol{D}\left(I_{Z}\right)\right)$. Pick $w \in \boldsymbol{h}^{i}\left(\boldsymbol{D}\left(A^{\bullet}\right)\right)$ such that $\alpha_{i}(z)=\gamma_{i}(w)$. Since $\delta_{i}\left(\alpha_{i}(z)\right)=0$ and $\Phi^{i}$ is injective, it follows that there exists a $u \in \boldsymbol{h}^{i-1}\left(\underline{\omega}_{X, Z}^{\bullet}\right)$ such that $\beta_{i}(u)=w$. Therefore, $\alpha_{i}\left(\Phi^{i-1}(u)\right)=\alpha_{i}(z)$ and so

$$
z-\Phi^{i-1}(u) \in f \cdot \boldsymbol{h}^{i-1}\left(\boldsymbol{D}\left(I_{Z}\right)\right) .
$$

Now, fix $E_{i-1}$ to be the cokernel of $\Phi^{i-1}$ and set $\bar{z} \in E_{i-1}$ to be the image of $z$. Equation (4.2.2) then guarantees that $\bar{z} \in f \cdot E_{i-1}$. The multiplication map $E_{i-1} \stackrel{\times f}{\longrightarrow} E_{i-1}$ is then surjective and so Nakayama's lemma guarantees that $\Phi^{i-1}$ is 
also surjective. Therefore $\underline{\omega}_{X, Z}^{*} \rightarrow \boldsymbol{D}\left(I_{Z}\right)$ is a quasi-isomorphism, which implies that $(X, Z)$ is a Du Bois pair.

Corollary 4.3. Let $f: X \rightarrow B$ be a flat proper family of varieties over a smooth one-dimensional scheme, $B$ being essentially of finite type over $\mathbb{C}$ ( for instance, a smooth curve). Further, let $Z \subseteq X$ be a subscheme such that no component of $Z$ is contained in any component of any fiber of $f$ and $b \in B$ a closed point such that $\left(X_{b}, Z_{b}\right)$ is a Du Bois pair. Then there exists a neighborhood $b \in U \subseteq B$ such that

(a) $(X, Z)$ is Du Bois over $U$, and

(b) the fibers $\left(X_{u}, Z_{u}\right)$ are Du Bois for all $u \in U$.

Proof. The non-Du Bois locus $T$ of $(X, Z)$ is closed, and since $f$ is proper, $f(T)$ is also closed. Hence (a) follows from Theorem 4.2 and by replacing $B$ with an open set, we may assume that $(X, Z)$ is Du Bois. Then the Bertini-type theorem Lemma 2.17(b) implies that (b) follows after possibly shrinking $U$.

Corollary 4.4. Let $f: X \rightarrow B$ be a flat proper family of varieties over a smooth scheme $B$ essentially of finite type over $\mathbb{C}$. Further let $Z \subseteq X$ be a subscheme which is also flat over $B$ and $b \in B$ a closed point such that $\left(X_{b}, Z_{b}\right)$ is a Du Bois pair. Then there exists a neighborhood $U \subseteq B, b \in U$, such that $(X, Z)$ is Du Bois over $U$.

Proof. We may assume that $B$ is affine and let $d=\operatorname{dim} B$. We first show that $(X, Z)$ itself is Du Bois in a neighborhood of $\left(X_{b}, Z_{b}\right)$. Let $H_{1}, \ldots, H_{d}$ be general smooth subschemes going through $b$ whose local defining equations generate the maximal ideal of $b$ (i.e., locally analytically they are coordinate hyperplanes). The pair $\left(X_{b}, Z_{b}\right)=\left(X_{H_{1} \cap H_{2} \cap \cdots \cap H_{d}}, Z_{H_{1} \cap H_{2} \cap \cdots \cap H_{d}}\right)$ is Du Bois by assumption, hence since $X_{b}=X_{H_{1} \cap \cdots \cap H_{d}}$ is a hypersurface in $X_{H_{2} \cap \cdots \cap H_{d}}$ it follows that the pair $\left(X_{H_{2} \cap \cdots \cap H_{d}}, Z_{H_{2} \cap \cdots \cap H_{d}}\right)$ is Du Bois in a neighborhood of $X_{b}$, by Corollary 4.3. Let $W_{1}$ denote the non-Du Bois locus of $\left(X_{H_{2} \cap \cdots \cap H_{d}}, Z_{H_{2} \cap \cdots \cap H_{d}}\right)$. Since $W_{1}$ is closed and $f$ is proper, we see that $f\left(W_{1}\right)$ is closed in $H_{2} \cap \cdots \cap H_{d}$ and doesn't contain $b$. Shrinking $B$ if necessary, we may assume that $W_{1}$ is empty. Next observe that $H_{2} \cap \cdots \cap H_{d}$ is a hypersurface in $H_{3} \cap \cdots \cap H_{d}$ and so again we see that $\left(X_{H_{3} \cap \cdots \cap H_{d}}, Z_{H_{3} \cap \cdots \cap H_{d}}\right)$ is Du Bois in a neighborhood of $X_{H_{2} \cap \cdots \cap H_{d}}$ by Corollary 4.3. Set $W_{2}$ to be the non-Du Bois locus of $\left(X_{H_{3} \cap \cdots \cap H_{d}}, Z_{H_{3} \cap \cdots \cap H_{d}}\right)$ and note that $f\left(W_{2}\right)$ does not intersect $H_{2} \cap \cdots \cap H_{d}$. We shrink $B$ again if necessary so that $W_{2}=\varnothing$. Iterating this procedure proves the statement.

In order to extend Corollary 4.3(b) to families over arbitrary-dimensional bases we need the following lemma.

Lemma 4.5. Let $f: X \rightarrow B$ be a flat proper family of varieties over a scheme $B$ essentially of finite type over $\mathbb{C}$. Further, let $Z \subseteq X$ be a subscheme which is also 
flat over $B$ and assume that $(X, Z)$ is a Du Bois pair. Then

$$
V=\left\{b \in B \mid\left(X_{b}, Z_{b}\right) \text { is a Du Bois pair }\right\}
$$

is a constructible set in B. Furthermore, if B is smooth, then $V$ is open.

Proof. We use induction on the dimension of $B$.

Let $\pi: B^{\prime} \rightarrow B$ be a resolution of singularities and consider the base change $f^{\prime}: X^{\prime}=X_{B^{\prime}} \rightarrow B^{\prime}$, assumed to be a flat proper family over $B^{\prime}$ and with $Z^{\prime}=Z_{B^{\prime}} \subseteq X^{\prime}$ a subscheme that is flat over $B^{\prime}$. Notice that all the fibers of $f^{\prime}: X^{\prime} \rightarrow B^{\prime}$ appear as fibers of $f: X \rightarrow B$ (up to harmless field extension), so $b^{\prime} \in V^{\prime}=\pi^{-1}(V) \subseteq B^{\prime}$ if and only if the fiber $\left(X_{b^{\prime}}^{\prime}, Z_{b^{\prime}}^{\prime}\right)$ is a Du Bois pair. It follows from Corollary 4.4 that by replacing $B^{\prime}$ with an open subset we may assume that $\left(X^{\prime}, Z^{\prime}\right)$ is a Du Bois pair. It also follows that it is enough to prove the statement over a smooth irreducible base. Indeed, that implies that $V^{\prime}$ is open in $B^{\prime}$ and hence $V=f\left(V^{\prime}\right)$ is constructible.

To simplify notation we will replace $B$ with $B^{\prime}$ and assume that $B$ is smooth and irreducible, but use the inductive hypothesis without these additional assumptions.

The Bertini-type statement Lemma 2.17(b) implies that, if $V \neq \varnothing$, there is a dense open subset $U \subseteq B$ contained in $V$. The case $\operatorname{dim} B=1$ follows immediately via the fact that in a curve any set containing a dense open set is itself open.

In general, it follows that $\operatorname{dim}(B \backslash U)<\operatorname{dim} B$ so by induction $V \backslash U$ is a constructible set in $B \backslash U$ and hence $V$ is constructible in $B$. In the case of a smooth base Corollary 4.4 implies that $V$ is stable under generalization and since we have just proved that it is constructible it follows that it is open.

Corollary 4.6. Let $f: X \rightarrow B$ be a flat proper family of varieties over a smooth scheme $B$ essentially of finite type over $\mathbb{C}$. Further let $Z \subseteq X$ be a subscheme which is also flat over $B$ and $b \in B$ a closed point such that $\left(X_{b}, Z_{b}\right)$ is a Du Bois pair. Then there exists a neighborhood $U \subseteq B, b \in U$, such that $\left(X_{u}, Z_{u}\right)$ is a Du Bois pair for all $u \in U$.

Proof. Observe that the non-Du Bois locus $W$ of $(X, Z)$ is closed in $X$ and since $f$ is proper, $f(W)$ is also closed in $B$. Note that $f(W)$ does not contain $b$ so it also does not contain the generic point of $B$. Hence by replacing $B$ by a neighborhood $U \subseteq B$ of $b \in B$, we may assume that $(X, Z)$ is Du Bois. Then the statement follows from Lemma 4.5.

Remark 4.7. One can recover special cases of inversion of adjunction for $\log$ canonicity [Kawakita 2007] easily from Theorem 4.2. For instance, let $(X, D+H)$ be a pair with $K_{X}, D$ and $H$ Cartier and assume that $\left(H,\left.D\right|_{H}\right)$ is slc or equivalently Du Bois [Kollár 2013]. Then $(X, D+H)$ is Du Bois or equivalently lc by Theorem 4.2. 


\section{Generalizing the Kollár-Kovács result to pairs}

We recall Theorem 7.12 of [Kollár and Kovács 2010]. Let $f: X \rightarrow B$ be a flat projective family of varieties with Du Bois singularities. Then if $B$ is connected and the general fiber is Cohen-Macaulay, then all the fibers are Cohen-Macaulay.

We would like to generalize this to the context of Du Bois pairs, at least in the case when $Z$ is a divisor. We recommend the reader have a copy of [Kollár and Kovács 2010] available when reading this section as we refer to a number of lemmas therein. We begin by generalizing a result of Du Bois and Jarraud to pairs; cf. [Du Bois and Jarraud 1974; Du Bois 1981, théorème 4.6].

Theorem 5.1. Let $f: X \rightarrow B$ be a flat proper morphism between schemes of finite type over $\mathbb{C}$. Assume that $B$ is smooth and let $Z \subseteq X$ be a subscheme that is flat over B. Further assume that the geometric fibers $\left(X_{b}, Z_{b}\right) \rightarrow b$ are Du Bois. Then for all $i, R^{i} f_{*} \mathscr{I}_{Z}$ is locally free of finite rank and compatible with base change; in other words $\left(\mathcal{R}^{i} f_{*} \mathscr{I}_{Z}\right)_{T} \simeq \mathcal{R}^{i} f_{*} \mathscr{I}_{Z_{T}}$ for any morphism $T \rightarrow B$.

Proof. For some $b \in B$, let $\mathfrak{m}$ be the maximal ideal of $\mathscr{O}_{B, b}$ and $S=S_{n}=$ $\operatorname{Spec} \mathscr{O}_{B, b} / \mathfrak{m}^{n+1}$ for $n \in \mathbb{N}$. Further, let $\mathscr{I}_{Z_{b}}$ and $\mathscr{I}_{Z_{S}}$ denote the ideal sheaves of $Z_{b}$ in $X_{b}$ and $Z_{S}$ in $X_{S}$, respectively. Consider the commutative diagram

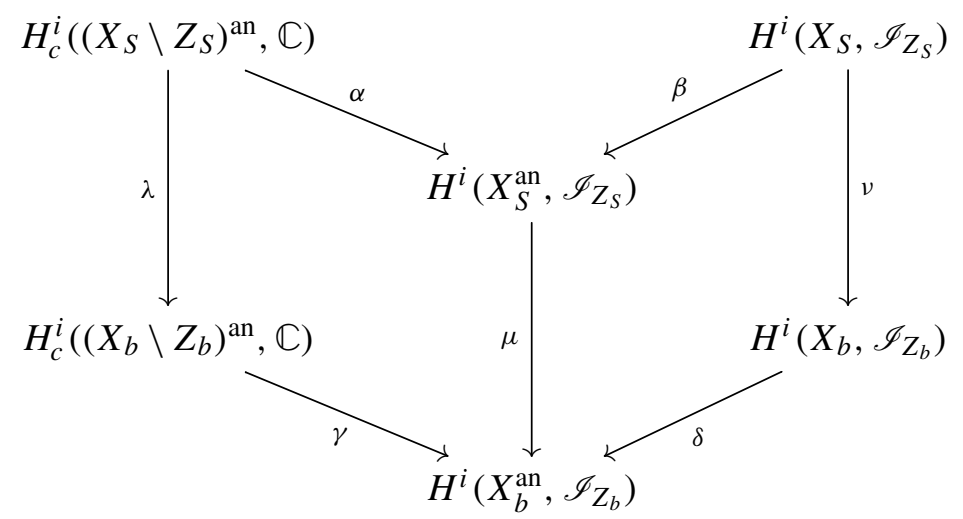

Observe that $\lambda$ is an isomorphism since $X_{S}$ and $X_{b}$ have the same support. By [Kovács 2011a, Theorem 4.1], cf. [Kollár 2013, Theorem 6.8], $\gamma$ is surjective, so $\gamma \circ \lambda=\mu \circ \alpha$ is surjective and hence $\mu$ is surjective. By Serre's GAGA principle [Serre 1956], $\beta$ and $\delta$ are isomorphisms and hence $v$ is surjective. Finally, the statement follows by cohomology and base change [Grothendieck 1963, §7.7].

Next we prove the analogue of the main flatness and base change result of Kollár and Kovács [2010, Theorem 7.9] for Du Bois pairs.

Theorem 5.2. Let $f: X \rightarrow B$ be a flat projective morphism between schemes of finite type over $\mathbb{C}$, and assume that $B$ is smooth. Let $Z \subseteq X$ be a closed subscheme 
that is flat over $B$ and $\mathscr{L}$ a relatively ample line bundle on $X$. Assume $(X, Z)$ is Du Bois. Then:

(a) The sheaves $\boldsymbol{h}^{-i}\left(\mathcal{R} \mathcal{H o m}_{\mathscr{O}_{X}}\left(\mathscr{I}_{Z}, \omega_{f}^{\bullet}\right)\right)$ are flat over $B$ for all $i$.

(b) The sheaves $f_{*}\left(\boldsymbol{h}^{-i}\left(\mathcal{R} \mathcal{H}_{0} m_{\mathscr{O}_{X}}\left(\mathscr{I}_{Z}, \omega_{f}^{*}\right)\right) \otimes \mathscr{L}^{q}\right)$ are locally free and compatible with arbitrary base change for all $i>0$ and $q \gg 0$.

(c) For any base change $\vartheta: T \rightarrow B$ and for all $i>0$,

$$
\left(\boldsymbol{h}^{-i}\left(\mathcal{R} \mathcal{H o m}_{\mathscr{O}_{X}}\left(\mathscr{I}_{Z}, \omega_{f}^{\cdot}\right)\right)\right)_{T} \simeq \boldsymbol{h}^{-i}\left(\mathcal{R} \mathcal{H o m}_{\mathscr{O}_{X}}\left(\mathscr{I}_{Z_{T}}, \omega_{f_{T}}\right)\right) .
$$

Proof. We follow the proof of [Kollár and Kovács 2010, Theorem 7.9]. We may assume that $B=\operatorname{Spec} R$ is affine and hence that $\mathscr{L}^{m}$ is globally generated for $m \gg 0$. For such an $m \gg 0$, choose a general section $\sigma \in H^{0}\left(X, \mathscr{L}^{m}\right)$ and consider the cyclic cover induced by $\sigma$ :

$$
\mathscr{A}=\bigoplus_{j=0}^{m-1} \mathscr{L}^{-j} \simeq \bigoplus_{j=0}^{m-1} \mathscr{L}^{-j} t^{j} /\left(t^{m}-\sigma\right) .
$$

Set $h: Y=\operatorname{Spec}_{X} \mathscr{A} \rightarrow X$, and $Z_{Y}=h^{-1} Z$ with the induced reduced scheme structure. Then the geometric fibers of the composition $\left(Y, Z_{Y}\right) \rightarrow B$ are also Du Bois by [Kollár 2013, Corollary 6.21]. Note that by construction $\mathscr{I}_{Z_{Y}}=$ $\bigoplus_{j=0}^{m-1} \mathscr{I}_{Z} \otimes \mathscr{L}^{-j}$. Hence $\mathcal{R}^{i} h_{*} \mathscr{I}_{Z_{Y}}$ is locally free of finite rank and compatible with arbitrary base change by Theorem 5.1. It follows that the summands of these modules, the $\mathcal{R}^{i} f_{*}\left(\mathscr{I}_{Z} \otimes \mathscr{L}^{-j}\right)$, are also locally free and compatible with base change. Since we may choose $m$ arbitrarily large, this holds for all $j \in \mathbb{N}$. It follows immediately that $\mathcal{H o m}_{\mathscr{O}_{B}}\left(\mathcal{R}^{i} f_{*}\left(\mathscr{I}_{Z} \otimes \mathscr{L}^{-j}\right), \mathscr{O}_{B}\right)$ is also locally free and compatible with base change.

By Grothendieck duality and [Kollár and Kovács 2010, Lemma 7.3] (see the proof of Lemma 7.2 in that paper follows that

$$
\begin{aligned}
\mathcal{H o m}_{\mathscr{O}_{B}}\left(\mathcal{R}^{i} f_{*}\left(\mathscr{I}_{Z} \otimes \mathscr{L}^{-q}\right), \mathscr{O}_{B}\right) & \simeq f_{*} \boldsymbol{h}^{-i}\left(\mathcal{R} \mathcal{H o m}_{\mathscr{O}_{X}}\left(\mathscr{I}_{Z}, \omega_{f}^{\bullet} \otimes \mathscr{L}^{q}\right)\right) \\
& \simeq f_{*}\left(\boldsymbol{h}^{-i}\left(\mathcal{R} \mathcal{H o m}_{\mathscr{O}_{X}}\left(\mathscr{I}_{Z}, \omega_{f}^{\bullet}\right) \otimes \mathscr{L}^{q}\right)\right)
\end{aligned}
$$

and hence (b) is proven. Just as in [Kollár and Kovács 2010, Theorem 7.9], (a) follows from (b) by an argument similar to [Hartshorne 1977, Chapter III, Theorem 9.9].

Finally we prove (c). Since $f: X \rightarrow B$ is projective and $B$ is affine, we may factor $f$ as $X \stackrel{i}{\rightarrow} \mathbb{P}_{B}^{n} \stackrel{\pi}{\rightarrow} B$. It then suffices to show that

$$
\varrho^{-i}:\left(\boldsymbol{h}^{-i}\left(\mathcal{R} \mathcal{H o m}_{\mathscr{O}_{\mathbb{P}_{B}^{n}}}\left(\mathscr{I}_{Z}, \omega_{\pi}[n]\right)\right)\right)_{T} \rightarrow \boldsymbol{h}^{-i}\left(\mathcal{R} \mathcal{H o m}_{\mathscr{O}_{T}^{n}}\left(\mathscr{I}_{Z_{T}}, \omega_{\pi}[n]\right)\right)
$$


is an isomorphism. As in [Kollár and Kovács 2010, Theorem 7.9], we proceed by descending induction on $i$ (the base case where $i \gg 0$ is obvious). We observe that $\mathscr{I}_{Z}$ is flat since so are $\mathscr{O}_{X}$ and $\mathscr{O}_{Z}$ and assume that $\varrho^{-(i+1)}$ is an isomorphism by induction. Since $\boldsymbol{h}^{-i}\left(\mathcal{R} \mathcal{H o m}_{\mathscr{O}_{\mathbb{P}}^{n}}\left(\mathscr{I}_{Z}, \omega_{\pi}[n]\right)\right)$ is flat, by (a), we may apply [Altman and Kleiman 1980, Theorem 1.9], which completes the proof.

The following is the analog of [Kollár and Kovács 2010, Theorem 7.11] for pairs.

Theorem 5.3. Let $f: X \rightarrow B$ be a flat projective morphism between schemes of finite type over $\mathbb{C}$. Assume that $B$ is smooth and let $Z \subseteq X$ be a subscheme that is flat over B. Let $x \in X$ be a closed point and let $b=f(x)$. Then $\mathscr{I}_{Z_{b}} \subseteq \mathscr{O}_{X_{b}}$ is $\mathrm{S}_{k}$ at $x$ if and only if

$$
\left(\boldsymbol{h}^{-i}\left(\mathcal{R} \mathcal{H o m}_{\mathscr{O}_{X}}\left(\mathscr{I}_{Z}, \omega_{f}^{\bullet}\right)\right)\right)_{y}=0
$$

for $i<\min \left(k+\operatorname{dim}\{\bar{y}\}, \operatorname{dim}_{x} X\right)$ and for all $y \in X_{b}$ such that $x \in\{\bar{y}\}$. In particular, $\mathscr{I}_{Z_{b}}$ is $\mathrm{S}_{k}$ if and only if (5.3.1) holds for $i<\min \left(k+\operatorname{dim}\left\{\overline{y\}}, \operatorname{dim}_{x} X\right)\right.$ and for all $y \in X_{b}$ (not restricted to closed points).

First we prove a lemma.

Lemma 5.4. Let $X$ be a scheme that admits a dualizing complex $\omega_{X}^{\bullet}$. Let $x \in X$ and let $\mathscr{F}$ be a coherent sheaf on $X$. Then $\mathscr{F}$ is $\mathrm{S}_{k}$ at $x \in X$ if and only if

$$
\left(\boldsymbol{h}^{-i}\left(\mathcal{R} \mathcal{H o m}_{\mathscr{O}_{X}}\left(\mathscr{F}, \omega_{X}^{\bullet}\right)\right)\right)_{y}=0
$$

for $i<\min \left(k, \operatorname{dim} \mathscr{F}_{y}\right)+\operatorname{dim}\{\overline{y y}$ and for all $y \in X$ such that $x \in\{\bar{y}\}$.

Proof. This is a consequence of local duality [Hartshorne 1966] and the cohomological criterion for depth; see for instance [Kovács 2011b, Proposition 3.2].

Proof of Theorem 5.3. By the lemma, $\mathscr{I}_{Z_{b}} \subseteq \mathscr{O}_{X_{b}}$ is $\mathrm{S}_{k}$ at $x$ if and only if

$$
\left(\boldsymbol{h}^{-i}\left(\mathcal{R} \mathcal{H o m}_{\mathscr{O}_{X_{b}}}\left(\mathscr{I}_{Z_{b}}, \omega_{X_{b}}^{\cdot}\right)\right)\right)_{y}=0
$$

for $i<\min \left(k, \operatorname{dim}\left(\mathscr{I}_{Z_{b}}\right)_{y}\right)+\operatorname{dim}\left\{\overline{y\}}=\min \left(k+\operatorname{dim}\left\{\overline{y\}}, \operatorname{dim}_{x} X\right)\right.\right.$ and for all $y \in X_{b}$ such that $x \in\{\bar{y}\}$. By Theorem 5.2,

$$
\begin{aligned}
\left(\boldsymbol{h}^{-i}\left(\mathcal{R} \mathcal{H o m}_{\mathscr{O}_{X_{b}}}\left(\mathscr{I}_{Z_{b}}, \omega_{X_{b}}\right)\right)\right)_{y} & \simeq\left(\left(\boldsymbol{h}^{-i}\left(\mathcal{R} \mathcal{H o m}_{\mathscr{O}_{X}}\left(\mathscr{I}_{Z}, \omega_{f}^{\bullet}\right)\right)_{b}\right)_{y}\right. \\
& \simeq\left(\left(\boldsymbol{h}^{-i}\left(\mathcal{R} \mathcal{H o m}_{\mathscr{O}_{X}}\left(\mathscr{I}_{Z}, \omega_{f}^{\bullet}\right)\right)_{y}\right)_{b} .\right.
\end{aligned}
$$

But notice that the right side is zero if and only if $\left(\boldsymbol{h}^{-i}\left(\mathcal{R} \mathcal{H o m}_{\mathscr{O}_{X}}\left(\mathscr{I}_{Z}, \omega_{f}^{\bullet}\right)\right)\right)_{y}$ is zero by Nakayama's lemma. This implies the desired statement.

Finally, we describe how the $S_{k}$ condition behaves for pairs in families where the fibers are Du Bois. 
Theorem 5.5. Let $f:(X, Z) \rightarrow B$ be a flat projective family with $\mathscr{O}_{Z}$ (and hence $\left.\mathscr{I}_{Z}\right)$ flat over $B$ as well. Assume that all the fiber pairs $\left(X_{b}, Z_{b}\right)$ are Du Bois. Assume also that $B$ is connected and the generic fibers $\left(\mathscr{I}_{Z}\right)_{\text {gen }}$ are $S_{k}$. Then all the fibers $\left(\mathscr{I}_{Z}\right)_{b}$ are $\mathrm{S}_{k}$.

Proof. By working with one component of $B$ at a time, we may assume that $B$ is irreducible and hence that $X$ is equidimensional. If $\left(\mathscr{I}_{Z}\right)_{b} \simeq \mathscr{I}_{Z_{b}}$ (by flatness of $\mathscr{O}_{Z}$ ) is not $\mathrm{S}_{k}$ at some point $y \in X_{b}$, then by Theorem $5.3, \boldsymbol{h}^{-i}\left(\mathcal{R} \mathcal{H o m}_{\mathscr{O}_{X}}\left(\mathscr{I}_{Z}, \omega_{f}^{\bullet}\right)\right) \neq 0$ near $y$ for some $i<\min (k+\operatorname{dim}\{\overline{y y}$, $\operatorname{dim} X)$. Fix an irreducible component $W \subseteq \operatorname{supp}\left(\boldsymbol{h}^{-i}\left(\mathcal{R} \mathcal{H o m}_{\mathscr{O}_{X}}\left(\mathscr{I}_{Z}, \omega_{f}^{\bullet}\right)\right)\right)$ and observe that $\operatorname{dim} W_{b}$ is constant for $b \in B$ since $\boldsymbol{h}^{-i}\left(\mathcal{R} \mathcal{H o m}_{\mathscr{O}_{X}}\left(\mathscr{I}_{Z}, \omega_{f}^{*}\right)\right)$ is flat by Theorem 5.2(a). However, in that case it follows that $\boldsymbol{h}^{-i}\left(\mathcal{R} \mathcal{H o m}_{\mathscr{O}_{X}}\left(\mathscr{I}_{Z}, \omega_{f}^{\bullet}\right)\right)$ is nonzero near some point $\eta \in X_{\text {gen }}$ such that $\operatorname{dim}\left\{\overline{\eta \eta}=\operatorname{dim}\left\{\overline{y\}}\right.\right.$, which contradicts the assumption that the generic fiber is $\mathrm{S}_{k}$ by Theorem 5.3.

Corollary 5.6. Let $f:(X, Z) \rightarrow B$ be a flat projective family with $\mathscr{O}_{Z}$ (and hence $\left.\mathscr{I}_{Z}\right)$ flat over $B$ as well. Assume that all the fiber pairs $\left(X_{b}, Z_{b}\right)$ are Du Bois. Assume also that $B$ is connected and the generic fibers $\left(\mathscr{I}_{Z}\right)_{\text {gen }}$ are Cohen-Macaulay. Then all the fibers $\left(\mathscr{I}_{Z}\right)_{b}$ are Cohen-Macaulay.

At this point it is natural to ask the next question.

Question 5.7. Assume that $(X, Z)$ is a pair and that $H \subseteq X$ is a Cartier divisor such that $(H, Z \cap H)$ is a Du Bois pair. If $\left.\mathscr{I}_{Z}\right|_{X \backslash H}$ is Cohen-Macaulay, does it follow that $\mathscr{I}_{Z}$ is Cohen-Macaulay?

In the case that $Z=\varnothing$, the analogous result holds in characteristic $p>0$ for $F$-injective singularities by [Horiuchi et al. 2014, Appendix by K. Schwede and A. K. Singh].

\section{Generalizing Kovács-Schwede-Smith to pairs}

The goal of this section is to prove the analog of the main result of [Kovács et al. 2010] for pairs $(X, Z)$.

Lemma 6.1. Let $X$ be a normal d-dimensional variety, $Z \subsetneq X$ a reduced closed subscheme and $\Sigma \subsetneq X$ a codimension $\geq 2$ subset containing the singular locus of $X$. Let $\pi: \widetilde{X} \rightarrow X$ be a log resolution of $(X, \Sigma \cup Z)$ with $E=\pi^{-1}(\Sigma \cup Z)_{\mathrm{red}}$. Then

(a) $\underline{\Omega}_{X, \Sigma \cup Z}^{0} \simeq \mathcal{R} \pi_{*} \mathscr{O}_{\widetilde{X}}(-E)$, and

(b) $\boldsymbol{h}^{-d}\left(\underline{\omega}_{X, Z}^{\bullet}\right) \simeq \pi_{*} \omega_{\widetilde{X}}(E)$.

Proof. First we claim that both $\mathcal{R} \pi_{*} \mathscr{O}_{\widetilde{X}}(-E)$ and $\pi_{*} \omega_{\widetilde{X}}(E)$ are independent of the choice of $\pi$. This was proved for $\mathcal{R} \pi_{*} \mathscr{O} \widetilde{X}(-E)$ on pages 67-68 in the proof of [Kovács and Schwede 2011b, Theorem 6.4] and for $\pi_{*} \omega_{\widetilde{X}}(E)$ in [Kovács et al. 
2010, Lemma 3.12]. Therefore we are free to choose $\pi$ and hence we may assume that it is an isomorphism outside of $\Sigma \cup Z$. We have the distinguished triangle

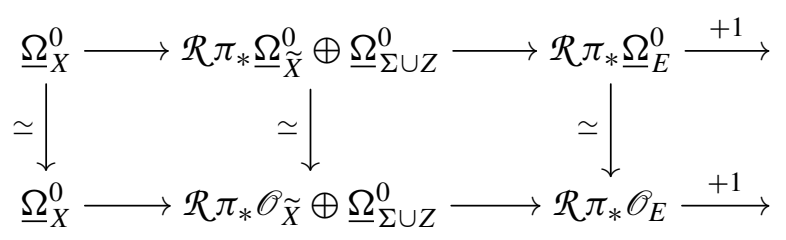

The isomorphisms follow since $\tilde{X}$ and $E$ are Du Bois. In the next diagram the first two rows are distinguished triangles by definition; see (2.7.1). The third row is simply the pushforward of a natural short exact sequence from $\tilde{X}$. The previous diagram and [Kollár and Kovács 2010, Lemma 2.1] (or simply the octahedral axiom) imply that $\alpha$ below is an isomorphism. The other two isomorphisms again follow since $\widetilde{X}$ and $E$ are Du Bois. Note that the columns are not exact.

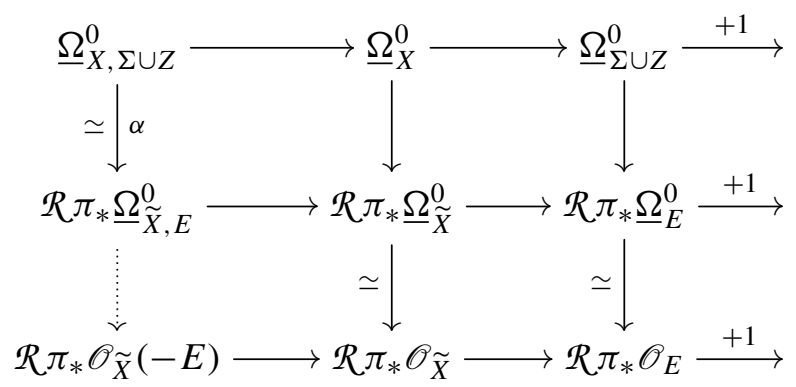

It follows that the dotted arrow, and hence its composition with $\alpha$, are also isomorphisms. This proves (a).

In order to prove (b), consider the map $\underline{\Omega}_{X, \Sigma \cup Z}^{0} \rightarrow \underline{\Omega}_{X, Z}^{0}$ obtained in

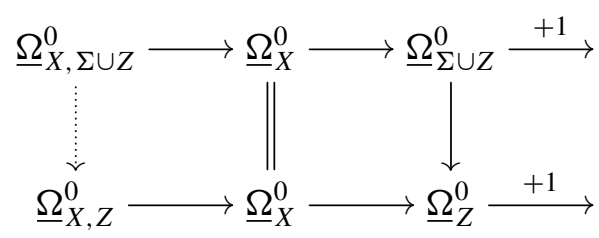

Now we have a distinguished triangle

$$
\underline{\Omega}_{X, \Sigma \cup Z}^{0} \rightarrow \underline{\Omega}_{X, Z}^{0} \rightarrow C \stackrel{+1}{\longrightarrow}
$$

Claim 6.2. With the above notation, $0=\boldsymbol{h}^{-d}\left(\boldsymbol{D}\left(C^{\bullet}\right)\right)=\boldsymbol{h}^{-d+1}\left(\boldsymbol{D}\left(C^{\bullet}\right)\right)$.

Proof of claim. Consider the following diagram with distinguished triangles as rows 
and columns:

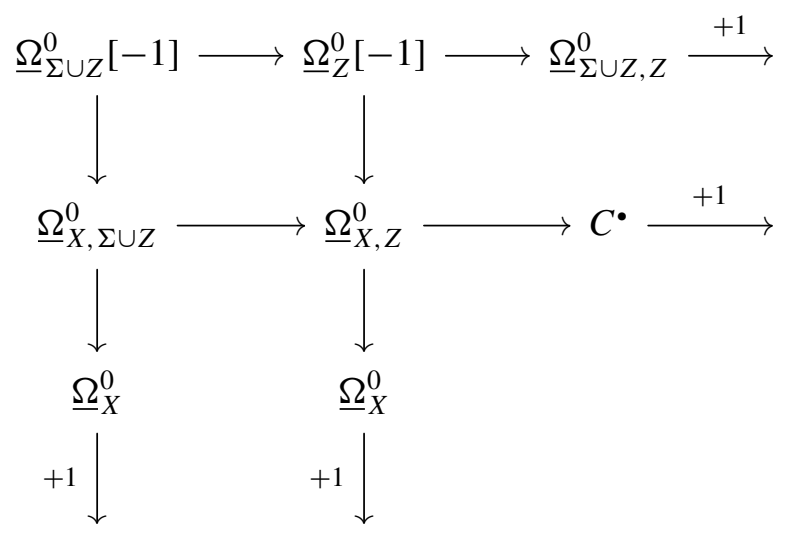

It follows from [Kovács 2013, Theorem B.1] that $C^{\bullet} \simeq \underline{\Omega}_{\Sigma \cup Z, Z}^{0}$. On the other hand, by Lemma $2.17(\mathrm{~d}), \underline{\Omega}_{\Sigma \cup Z, Z}^{0} \simeq \underline{\Omega}_{\Sigma, \Sigma \cap Z}^{0}$ and hence $C^{\bullet} \simeq \underline{\Omega}_{\Sigma, \Sigma \cap Z}^{0}$.

Next recall that by Theorem 3.2 there exists a natural injective map

$$
\boldsymbol{h}^{-j}\left(\boldsymbol{D}\left(\underline{\Omega}_{\Sigma, \Sigma \cap Z}^{0}\right)\right) \hookrightarrow \boldsymbol{h}^{-j}\left(\mathcal{R} \mathcal{H}{ }_{\dot{O}_{\Sigma}}^{\dot{O}_{\Sigma}}\left(\mathscr{I}_{(\Sigma \cap Z) \subseteq \Sigma}, \omega_{\Sigma}^{\bullet}\right)\right) .
$$

Since $\operatorname{dim} \Sigma \leq d-2$, the right hand side of (6.2.1) is zero for $j \geq d-1$, establishing the claim.

Grothendieck duality and part (a) imply that $\boldsymbol{h}^{-d}\left(\underline{\omega}_{X, \Sigma \cup Z}^{\bullet}\right) \simeq \pi_{*} \omega_{\widetilde{X}}(E)$ and it follows from Claim 6.2 that $\boldsymbol{h}^{-d}\left(\underline{\omega}_{X, \Sigma \cup Z}^{\cdot}\right) \simeq \boldsymbol{h}^{-d}\left(\underline{\omega}_{X, Z}^{\cdot}\right)$, which in turn implies part (b).

Theorem 6.3. Let $X$ be a normal variety and $Z \subseteq X$ a divisor. Let $\pi: \widetilde{X} \rightarrow X$ be a $\log$ resolution of $(X, Z)$ with $E=\pi^{-1}(Z)_{\mathrm{red}} \vee \operatorname{exc}(\pi)$. If $\mathscr{I}_{Z}$ is Cohen-Macaulay, then $(X, Z)$ is Du Bois if and only if

$$
\pi_{*} \omega_{\widetilde{X}}(E) \simeq \omega_{X}(Z) .
$$

Proof. Since $\mathscr{I}_{Z}$ is Cohen-Macaulay, $\mathcal{R} \mathcal{H o m}_{\mathscr{O}_{X}}\left(\mathscr{I}_{Z}, \omega_{X}^{\bullet}\right) \simeq \mathcal{H o m}_{\mathscr{O}_{X}}\left(\mathscr{I}_{Z}, \omega_{X}\right)[\operatorname{dim} X]$ by the local dual of the local cohomology criterion for Cohen-Macaulayness. Because the map

$$
\underline{\omega}_{X, Z}^{\bullet} \rightarrow \mathcal{R} \mathcal{H o m}_{\mathscr{O}_{X}}\left(\mathscr{I}_{Z}, \omega_{X}^{\bullet}\right)
$$

is injective on cohomology by Theorem 3.2, it follows that $\boldsymbol{h}^{i}\left(\underline{\omega}_{X, Z}^{\bullet}\right)=0$ for $i \neq-\operatorname{dim} X$ and hence $(X, Z)$ is Du Bois if and only if

$$
\boldsymbol{h}^{-\operatorname{dim} X}\left(\underline{\omega}_{X, Z}^{\cdot}\right) \rightarrow \mathcal{H o m}_{\mathscr{O}_{X}}\left(\mathscr{I}_{Z}, \omega_{X}\right) \simeq \omega_{X}(Z)
$$

is an isomorphism. But $\boldsymbol{h}^{-\operatorname{dim} X}\left(\underline{\omega}_{X, Z}^{\cdot}\right) \simeq \pi_{*} \omega_{\widetilde{X}}(E)$ by Lemma 6.1, so the statement follows. 


\section{An inversion of adjunction for rational and Du Bois pairs}

In this final section of the paper, we will prove the following theorem.

Theorem 7.1. Let $f: X \rightarrow B$ be a flat projective family with geometrically integral fibers over a smooth connected base $B, A \subseteq B$ a smooth closed subscheme containing no component of $B$ and $H=f^{-1}(A)=X \times_{B} A$ (with the induced scheme-theoretic structure). Let $D$ be a reduced codimension-1 subscheme of $X$ which is flat over B. Assume that for every $s \in A,\left(X_{s}, D_{s}\right)$ is Du Bois and that $(X \backslash H, D \backslash H)$ is a rational pair. Then $(X, D)$ is a rational pair.

Remark 7.2. In the introduction, $A$ was assumed to be a closed point. This version is more general and more convenient for our proof.

Remark 7.3. The assumptions also imply the following auxiliary conditions:

(a) Since $X \rightarrow B$ has geometrically integral fibers and $H$ is obtained by base change with a smooth subscheme, $H$ is reduced.

(b) $\mathscr{I}_{D}$ is flat over $B$ and no component of $D$ contains a fiber of $f$. In particular $D$ and $H$ have no common components.

(c) As for any $s \in A, H_{s}=X_{s}$, it follows that $(H, D \cap H)$ is Du Bois by Corollary 4.4.

(d) $X \backslash H$ is normal by the definition of a rational pair.

Before embarking on proving the theorem, we will first prove several lemmas that show that our situation is simpler than it might first appear.

First we show that we may assume that $A$ is a divisor in $B$.

Lemma 7.4. In order to prove Theorem 7.1 it is sufficient to assume that $A$ is a smooth Cartier divisor in $B$.

Proof. The statement is local over the base so we may assume that $B$ is affine. Additionally, since we only need to work in a neighborhood of a point $a \in A$, we may assume that $(X, D)$ is Du Bois and all the fibers $\left(X_{b}, D_{b}\right)$ for all $b \in B$ are Du Bois by Corollaries 4.4 and 4.6. Choose a general hypersurface $G$ containing $A$ and note that since $A$ is smooth we may assume that $G$ is smooth. Then the hypotheses of the theorem are satisfied for $G$ replacing $A$ as well since $X \backslash f^{-1}(G) \subseteq X \backslash f^{-1}(A)$ and since we already assumed that all the fibers $\left(X_{b}, D_{b}\right)$ over all points $b \in B$ were Du Bois.

From this point forward, we will assume that $B$ is a smooth affine scheme, $A$ is a smooth hypersurface in $B$ and $H=f^{*} A$.

Lemma 7.5. Under the assumptions of Theorem 7.1, $X$ is normal and thus $D$ is also a divisor. 
Proof. Since $H$ is reduced, every point $\eta \in H$ has depth at least $\min \left(1, \operatorname{dim} \mathscr{O}_{H, \eta}\right)$. Because $f: X \rightarrow B$ is flat, the local defining equation of $H$ is a regular element in $\mathscr{O}_{X}$, so any point $\eta \in X$ that lies in $H$ has depth at least $\min \left(2, \operatorname{dim} \mathscr{O}_{X, \eta}\right)$. Since $X \backslash H$ is normal it is $S_{2}$ and so $X$ is $S_{2}$ everywhere. Finally observe that $H$ is reduced, hence generically regular and $X \backslash H$ is $\mathrm{R}_{1}$. As $H$ is Cartier, this implies that $X$ is also $\mathrm{R}_{1}$ and therefore normal.

Now observe that the fact that $(H, D \cap H)$ is Du Bois (see Remark 7.3(b)) says something about the structure of $D$ on $X$.

Lemma 7.6. With notation as in Theorem 7.1, no stratum of the snc locus of $(X, D)$ can be contained inside $H$.

Proof. Assume to the contrary that there exists a stratum $Z$ of the snc locus of $(X, D)$ contained in $H$. Let $\eta$ be the generic point of $Z$. By assumption $\eta \in H$ and $(X, D)$ is snc at $\eta$, so $\mathscr{O}_{X, \eta}$ is a regular ring. Let $n=\operatorname{dim} \mathscr{O}_{X, \eta}$. Replace $X$ by Spec $\mathscr{O}_{X, \eta}$ and $H$ and $D$ by their pullbacks to this local scheme (in this step we lose projectivity, but we will not need that for now). Note that $D$ is now Cartier and in fact snc. Furthermore $D+H$ has $n+1$ irreducible components containing $\eta$, so $(X, D+H)$ cannot be Du Bois (or equivalently log canonical since $X$ is Gorenstein). But as we observed, $(H, D \cap H)$ is a Du Bois pair. Then by Theorem 4.2 again we see that $(X, D+H)$ is Du Bois as well. This is a contradiction.

Next we setup the notation for the proof of Theorem 7.1. Let $\Sigma$ denote the non-snc locus of $(X, D)$. Observe that as $X$ is normal and $D$ is a reduced divisor by Lemma 7.5, we have that $\operatorname{codim}_{X}(\Sigma) \geq 2$.

Additionally assume that $\pi: Y \rightarrow X$ is a $\log$ resolution of $(X, D \cup H \cup \Sigma)$ that simultaneously gives a thrifty resolution of $(X, D)$. To see that such a $\pi$ exists, first take a thrifty resolution $\left(U, D_{U}\right)$ of $(X, D)$ and then perform a log resolution of the scheme-theoretic preimages of $H$ and $\Sigma$ on $U$ (while keeping the strict transform $D_{U}$ snc). The result can be assumed to be a thrifty resolution of $(X, D)$ since the preimages of $\Sigma$ and $H$ do not contain any strata of $\left(U, D_{U}\right)$ by Lemma 7.6.

Set $\bar{H}$ and $\bar{D}$ to be the reduced total transforms of $H$ and $D$ respectively, set $D_{Y}$ to be the strict transform of $D$ and set $E$ to be $\left(\pi^{-1}(\Sigma)\right)_{\text {red. }}$.

Proof of Theorem 7.1. Clearly $(X, D)$ is a Du Bois pair and all the fibers $\left(X_{b}, D_{b}\right)$ are Du Bois by Corollaries 4.4 and 4.6 (possibly after shrinking the base $B$ around $A$ ). By Corollary 5.6, we know that $\mathscr{O}_{X}(-D)$ is Cohen-Macaulay. Thus by the local dual version of the local cohomological criterion for Cohen-Macaulayness, $\mathcal{R} \mathcal{H o m}_{\mathscr{O}_{X}}\left(\mathscr{O}_{X}(-D), \omega_{X}^{\bullet}\right)$ has cohomology only in one term. In particular,

$$
\begin{aligned}
\operatorname{RHom}_{\mathscr{O}_{X}}\left(\mathscr{O}_{X}(-D), \omega_{X}^{\bullet}\right) & \simeq \mathcal{H o m}_{\mathscr{O}_{X}}\left(\mathscr{O}_{X}(-D), \omega_{X}\right)[\operatorname{dim} X] \\
& \simeq \omega_{X}(D)[\operatorname{dim} X] .
\end{aligned}
$$


Therefore by Proposition 2.7 it suffices to show that $\omega_{X}(D) \simeq \pi_{*} \omega_{Y}\left(D_{Y}\right)$.

Next observe that $\left(H,\left.D\right|_{H}\right)$ is a Du Bois pair by Corollary 4.4 and hence by Lemma 2.18 we see that $(X, D \cup H)=(X, D+H)$ is a Du Bois pair.

Claim 7.7. With notation as above, $\pi_{*} \omega_{Y}\left(D_{Y} \vee \bar{H} \vee E\right) \simeq \pi_{*} \omega_{Y}\left(D_{Y}+\bar{H}\right)$.

Note that $D_{Y}+\bar{H}=D_{Y} \vee \bar{H}$ since the divisors have no common components. Proof of claim. The containment $\supseteq$ is obvious since $D$ and $H$ do not share a component (see Remark 7.3(a)), so choose $f \in \pi_{*} \omega_{Y}\left(D_{Y} \vee \bar{H} \vee E\right)$. We observe that

$$
\operatorname{div}_{Y}(f)+K_{Y}+D_{Y} \vee \bar{H} \vee E=\operatorname{div}_{Y}(f)+K_{Y}+D_{Y}+\bar{H} \vee E \geq 0 .
$$

Working on $U=Y \backslash \bar{H}=\pi^{-1}(X \backslash H)$ we see that $\operatorname{div}_{U}(f)+K_{U}+\left.D_{Y}\right|_{U}+\left.E\right|_{U} \geq 0$. But since $(X \backslash H, D \backslash H)$ is a rational pair,

$$
\pi_{*} \omega_{U}\left(\left.D_{Y}\right|_{U}\right)=\pi_{*} \omega_{U}\left(\left.D_{Y}\right|_{U}+E\right)=\omega_{X \backslash H}\left(\left.D\right|_{X \backslash H}\right),
$$

so $\operatorname{div}_{U}(f)+K_{U}+\left.D_{Y}\right|_{U}+\left.E\right|_{U} \geq 0$ is equivalent to $\operatorname{div}_{U}(f)+K_{U}+\left.D_{Y}\right|_{U} \geq 0$. Because the components of $E$ that lie over $H$ are also components of $\bar{H}$, it follows that $\operatorname{div}_{Y}(f)+K_{Y}+D_{Y}+\bar{H} \geq 0$, proving the claim.

By Lemma 6.1 we see that $\boldsymbol{h}^{-\operatorname{dim} X}\left(\underline{\omega}_{X, D+H}^{\cdot}\right) \simeq \pi_{*} \omega_{Y}\left(D_{Y} \vee \bar{H} \vee E\right)$, which agrees with $\pi_{*} \omega_{Y}\left(D_{Y}+\bar{H}\right)$ by the claim. Since $(X, D+H)$ is a Du Bois pair, $\boldsymbol{h}^{-\operatorname{dim} X}\left(\underline{\omega}_{X, D+H}^{\cdot}\right) \simeq \omega_{X}(D+H)$ and so in conclusion we have that

$$
\omega_{X}(D+H) \simeq \pi_{*} \omega_{Y}\left(D_{Y}+\bar{H}\right) .
$$

Twisting both sides by $-H$ and using the projection formula we see that

$$
\omega_{X}(D) \simeq \pi_{*} \omega_{Y}\left(D_{Y}-\left(\pi^{*} H-\bar{H}\right)\right) \subseteq \pi_{*} \omega_{Y}\left(D_{Y}\right),
$$

since $\pi^{*} H-\bar{H}$ is effective. But $\pi_{*} \omega_{Y}\left(D_{Y}\right) \subseteq \omega_{X}(D)$ for any normal pair $(X, D)$ and so $\omega_{X}(D) \simeq \pi_{*} \omega_{Y}\left(D_{Y}\right)$ as desired.

Setting $D=0$ we obtain the following.

Corollary 7.8. Let $f: X \rightarrow B$ be a flat projective family over a smooth base $B$ and $H=f^{-1}(0)$ a special fiber. Assume that $H$ has Du Bois singularities and that $X \backslash H$ has rational singularities. Then $X$ has rational singularities.

There is a variant of our inversion of adjunction theorem that we would also like to prove (even in the $D=0$ case).

Conjecture 7.9. Assume $(X, D)$ is a pair with $D$ a reduced Weil divisor. Further assume that $H$ is a Cartier divisor on $X$, not having any components in common with $D$, such that $(H, D \cap H)$ is Du Bois and such that $(X \backslash H, D \backslash H)$ is a rational pair. Then $(X, D)$ is a rational pair. 
The only place where our proof above does not work in this situation is when we prove that $\mathscr{O}_{X}(-D)$ is Cohen-Macaulay. In particular, to accomplish this generalization, we would simply need a version of Corollary 5.6 that is not tied to a projective or proper family. What is missing is exactly a positive answer to Question 5.7.

\section{Acknowledgements}

The authors thank Zsolt Patakfalvi for several useful discussions and the referee for numerous helpful comments and corrections.

\section{References}

[Altman and Kleiman 1980] A. B. Altman and S. L. Kleiman, "Compactifying the Picard scheme", Adv. Math. 35:1 (1980), 50-112. MR Zbl

[Du Bois 1981] P. Du Bois, "Complexe de de Rham filtré d'une variété singulière”, Bull. Soc. Math. France 109: 1 (1981), 41-81. MR Zbl

[Du Bois and Jarraud 1974] P. Du Bois and P. Jarraud, "Une propriété de commutation au changement de base des images directes supérieures du faisceau structural”, C. R. Acad. Sci. Paris Sér. A 279 (1974), 745-747. MR Zbl

[Elkik 1978] R. Elkik, "Singularités rationnelles et déformations", Invent. Math. 47:2 (1978), 139147. MR Zbl

[Erickson 2014] L. Erickson, "Deformation invariance of rational pairs", preprint, 2014. arXiv

[Esnault 1990] H. Esnault, "Hodge type of subvarieties of $\mathbb{P}^{n}$ of small degrees", Math. Ann. 288:3 (1990), 549-551. MR Zbl

[Fedder and Watanabe 1989] R. Fedder and K.-I. Watanabe, "A characterization of $F$-regularity in terms of F-purity", pp. 227-245 in Commutative algebra (Berkeley, CA, 1987), edited by M. Hochster et al., Mathematical Sciences Research Institute Publications 15, Springer, New York, NY, 1989. MR Zbl

[Graf and Kovács 2014] P. Graf and S. J. Kovács, "Potentially Du Bois spaces”, J. Singul. 8 (2014), 117-134. MR Zbl

[Grauert and Riemenschneider 1970] H. Grauert and O. Riemenschneider, "Verschwindungssätze für analytische Kohomologiegruppen auf komplexen Räumen”, Invent. Math. 11 (1970), 263-292. MR $\mathrm{Zbl}$

[Greb et al. 2011] D. Greb, S. Kebekus, S. J. Kovács, and T. Peternell, "Differential forms on log canonical spaces”, Publ. Math. Inst. Hautes Études Sci. 114 (2011), 87-169. MR Zbl

[Grothendieck 1963] A. Grothendieck, "Éléments de géométrie algébrique, III: Étude cohomologique des faisceaux cohérents, II”, Inst. Hautes Études Sci. Publ. Math. 17 (1963), 5-91. MR Zbl

[Hacon 2014] C. D. Hacon, "On the log canonical inversion of adjunction”, Proc. Edinb. Math. Soc. (2) 57:1 (2014), 139-143. MR Zbl

[Hacon and Xu 2013] C. D. Hacon and C. Xu, "Existence of log canonical closures", Invent. Math. 192:1 (2013), 161-195. MR Zbl

[Hartshorne 1966] R. Hartshorne, Residues and duality, Lecture Notes in Mathematics 20, Springer, Berlin, 1966. MR Zbl 
[Hartshorne 1977] R. Hartshorne, Algebraic geometry, Graduate Texts in Mathematics 52, Springer, New York, NY, 1977. MR Zbl

[Horiuchi et al. 2014] J. Horiuchi, L. E. Miller, and K. Shimomoto, "Deformation of $F$-injectivity and local cohomology", Indiana Univ. Math. J. 63:4 (2014), 1139-1157. MR Zbl

[Karu 2000] K. Karu, "Minimal models and boundedness of stable varieties", J. Algebraic Geom. 9:1 (2000), 93-109. MR Zbl

[Kawakita 2007] M. Kawakita, "Inversion of adjunction on log canonicity”, Invent. Math. 167:1 (2007), 129-133. MR Zbl

[Kollár 1997] J. Kollár, "Singularities of pairs”, pp. 221-287 in Algebraic geometry (Santa Cruz, 1995), edited by J. Kollár et al., Proceedings of Symposia in Pure Mathematics 62:1, American Mathematical Society, Providence, RI, 1997. MR Zbl

[Kollár 2013] J. Kollár, Singularities of the minimal model program, Cambridge Tracts in Mathematics 200, Cambridge University Press, 2013. MR Zbl

[Kollár and Kovács 2010] J. Kollár and S. J. Kovács, "Log canonical singularities are Du Bois", J. Amer. Math. Soc. 23:3 (2010), 791-813. MR Zbl

[Kollár and Mori 1998] J. Kollár and S. Mori, Birational geometry of algebraic varieties, Cambridge Tracts in Mathematics 134, Cambridge University Press, 1998. MR Zbl

[Kollár and Shepherd-Barron 1988] J. Kollár and N. I. Shepherd-Barron, "Threefolds and deformations of surface singularities”, Invent. Math. 91:2 (1988), 299-338. MR Zbl

[Kovács 1999] S. J. Kovács, "Rational, log canonical, Du Bois singularities: on the conjectures of Kollár and Steenbrink”, Compos. Math. 118:2 (1999), 123-133. MR Zbl

[Kovács 2000] S. J. Kovács, "Rational, log canonical, Du Bois singularities, II: Kodaira vanishing and small deformations”, Compos. Math. 121:3 (2000), 297-304. MR Zbl

[Kovács 2011a] S. J. Kovács, "Du Bois pairs and vanishing theorems", Kyoto J. Math. 51:1 (2011), 47-69. MR Zbl

[Kovács 2011b] S. J. Kovács, "Irrational centers", Pure Appl. Math. Q. 7:4 (2011), 1495-1515. MR $\mathrm{Zbl}$

[Kovács 2013] S. J. Kovács, "Singularities of stable varieties", pp. 159-203 in Handbook of moduli, vol. 2, edited by G. Farkas and I. Morrison, Advanced Lectures in Mathematics 25, International Press, Somerville, MA, 2013. MR Zbl

[Kovács and Schwede 2011a] S. J. Kovács and K. Schwede, "Du Bois singularities deform”, preprint, 2011. To appear in Advanced Studies in Pure Mathematics. arXiv

[Kovács and Schwede 2011b] S. J. Kovács and K. Schwede, "Hodge theory meets the minimal model program: a survey of log canonical and Du Bois singularities", pp. 51-94 in Topology of stratified spaces, edited by G. Friedman et al., Mathematical Sciences Research Institute Publications 58, Cambridge University Press, 2011. MR Zbl

[Kovács et al. 2010] S. J. Kovács, K. Schwede, and K. E. Smith, "The canonical sheaf of Du Bois singularities", Adv. Math. 224:4 (2010), 1618-1640. MR Zbl

[Lunts 2012] V. A. Lunts, "Categorical resolutions, poset schemes, and Du Bois singularities", Int. Math. Res. Not. 2012:19 (2012), 4372-4420. MR Zbl

[Ma 2015] L. Ma, “F-injectivity and Buchsbaum singularities”, Math. Ann. 362:1-2 (2015), 25-42. MR Zbl

[Patakfalvi 2015] Z. Patakfalvi, "Semi-negativity of Hodge bundles associated to Du Bois families", J. Pure Appl. Algebra 219:12 (2015), 5387-5393. MR Zbl 
[Saito 2000] M. Saito, "Mixed Hodge complexes on algebraic varieties", Math. Ann. 316:2 (2000), 283-331. MR Zbl

[Schumacher 2012] G. Schumacher, "Positivity of relative canonical bundles and applications", Invent. Math. 190:1 (2012), 1-56. MR Zbl

[Schwede 2007] K. Schwede, "A simple characterization of Du Bois singularities", Compos. Math. 143:4 (2007), 813-828. MR Zbl

[Schwede 2009] K. Schwede, “F-adjunction”, Algebra Number Theory 3:8 (2009), 907-950. MR $\mathrm{Zbl}$

[Schwede and Takagi 2008] K. Schwede and S. Takagi, "Rational singularities associated to pairs", Michigan Math. J. 57 (2008), 625-658. MR Zbl

[Serre 1956] J.-P.. Serre, “Géométrie algébrique et géométrie analytique”, Ann. Inst. Fourier (Grenoble) 6 (1956), 1-42. MR Zbl

Communicated by Ravi Vakil

Received 2015-01-23 Revised 2016-03-17 Accepted 2016-04-27

skovacs@uw.edu

Department of Mathematics, University of Washington, Box 354350, Padelford C-138, Seattle, WA 98195, United States

schwede@math.utah.edu

Department of Mathematics, The University of Utah, 155 S 1400 E Room 233, Salt Lake City, UT 84112-0090, United States 


\section{Algebra \& Number Theory}

msp.org/ant

\section{EDITORS}

MANAGING EDITOR

Bjorn Poonen

Massachusetts Institute of Technology

Cambridge, USA

\author{
EDITORIAL BOARD CHAIR \\ David Eisenbud \\ University of California \\ Berkeley, USA
}

BOARD OF EDITORS

$\begin{aligned} \text { Dave Benson } & \text { University of Aberdeen, Scotland } & \text { Susan Montgomery } & \text { University of Southern California, USA } \\ \text { Richard E. Borcherds } & \text { University of California, Berkeley, USA } & \text { Shigefumi Mori } & \text { RIMS, Kyoto University, Japan } \\ \text { John H. Coates } & \text { University of Cambridge, UK } & \text { Raman Parimala } & \text { Emory University, USA } \\ \text { J-L. Colliot-Thélène } & \text { CNRS, Université Paris-Sud, France } & \text { Jonathan Pila } & \text { University of Oxford, UK } \\ \text { Brian D. Conrad } & \text { Stanford University, USA } & \text { Anand Pillay } & \text { University of Notre Dame, USA } \\ \text { Hélène Esnault } & \text { Freie Universität Berlin, Germany } & \text { Victor Reiner } & \text { University of Minnesota, USA } \\ \text { Hubert Flenner } & \text { Ruhr-Universität, Germany } & \text { Peter Sarnak } & \text { Princeton University, USA } \\ \text { Sergey Fomin } & \text { University of Michigan, USA } & \text { Joseph H. Silverman } & \text { Brown University, USA } \\ \text { Edward Frenkel } & \text { University of California, Berkeley, USA } & \text { Michael Singer } & \text { North Carolina State University, USA } \\ \text { Andrew Granville } & \text { Université de Montréal, Canada } & \text { Vasudevan Srinivas } & \text { Tata Inst. of Fund. Research, India } \\ \text { Joseph Gubeladze } & \text { San Francisco State University, USA } & \text { J. Toby Stafford } & \text { University of Michigan, USA } \\ \text { Roger Heath-Brown } & \text { Oxford University, UK } & \text { Ravi Vakil } & \text { Stanford University, USA } \\ \text { Craig Huneke } & \text { University of Virginia, USA } & \text { Michel van den Bergh } & \text { Hasselt University, Belgium } \\ \text { Kiran S. Kedlaya } & \text { Univ. of California, San Diego, USA } & \text { Marie-France Vignéras } & \text { Université Paris VII, France } \\ \text { János Kollár } & \text { Princeton University, USA } & \text { Kei-Ichi Watanabe } & \text { Nihon University, Japan } \\ \text { Yuri Manin } & \text { Northwestern University, USA } & \text { Efim Zelmanov } & \text { University of California, San Diego, USA } \\ \text { Philippe Michel } & \text { École Polytechnique Fédérale de Lausanne } & \text { Shou-Wu Zhang } & \text { Princeton University, USA }\end{aligned}$

PRODUCTION

production@msp.org

Silvio Levy, Scientific Editor

See inside back cover or msp.org/ant for submission instructions.

The subscription price for 2016 is US $\$ 290$ /year for the electronic version, and $\$ 485 /$ year (+\$55, if shipping outside the US) for print and electronic. Subscriptions, requests for back issues and changes of subscribers address should be sent to MSP.

Algebra \& Number Theory (ISSN 1944-7833 electronic, 1937-0652 printed) at Mathematical Sciences Publishers, 798 Evans Hall \#3840, c/o University of California, Berkeley, CA 94720-3840 is published continuously online. Periodical rate postage paid at Berkeley, CA 94704, and additional mailing offices.

ANT peer review and production are managed by EditFLow ${ }^{\circledR}$ from MSP.

\section{PUBLISHED BY}

- mathematical sciences publishers

nonprofit scientific publishing

http://msp.org/

() 2016 Mathematical Sciences Publishers 


\section{Algebra \& Number Theory}

Volume $10 \quad$ No. $5 \quad 2016$

Conjugacy classes of special automorphisms of the affine spaces

JÉRÉMY BLANC

Inversion of adjunction for rational and Du Bois pairs

SÁNDOR J. KOVÁCS and KARL SCHWEDE

Hochschild cohomology commutes with adic completion

LIRAN SHAUL

Bifurcations, intersections, and heights

LAURA DEMARCO

RANKEyA DATTA and KaREN E. SMITH

1091

Hoffmann's conjecture for totally singular forms of prime degree

STEPHEN SCULLY 